\title{
DIFFERENTIAL EQUATIONS ASSOCIATED WITH NONARITHMETIC FUCHSIAN GROUPS
}

\author{
IRENE I. BOUW AND MARTIN MÖLLER
}

\begin{abstract}
We describe globally nilpotent differential operators of rank 2 defined over a number field whose monodromy group is a nonarithmetic Fuchsian group. We show that these differential operators have an $\mathcal{S}$-integral solution. These differential operators are naturally associated with Teichmüller curves in genus 2. They are counterexamples to conjectures by Chudnovsky-Chudnovsky and Dwork. We also determine the field of moduli of primitive Teichmüller curves in genus 2, and an explicit equation in some cases.
\end{abstract}

Let $L$ be a Fuchsian differential operator of order 2 defined over a number field $K$. In the literature, one finds several conjectures which connect that $L$ "comes from geometry", is globally nilpotent, or admits an integral solution. Here "coming from geometry" could mean, for example, that $L$ is a direct factor of the Picard-Fuchs differential equation of a family of curves. The most famous of these is Grothendieck's $p$-curvature conjecture which says that every globally nilpotent differential equation comes from geometry. Another conjecture says that if $L$ admits an integral solution then $L$ comes from geometry. We refer to $\S[5$ for definitions, and to [1], [2], [6] and [15] for partial results and precise formulations of the conjectures.

In this paper, rather than proving a version of these conjectures, we show the existence of integral solutions of a certain interesting class of differential equations which come from geometry. Here a solution $u$ is integral if there exists a finite set of primes $\mathcal{S}$ such that the coefficients of $u$ are in the ring $\mathcal{O}_{\mathcal{S}} \subset K$ of $\mathcal{S}$-integral elements.

For hypergeometric differential equations the existence of an integral solution is well understood. Differential equations with 4 singularities which admit an integral solution are very rare. Zagier $([6],[17, \S 2.4)$ found in a huge computer search essentially only 6 of such differential operators with $\mathbb{Q}$-coefficients. All of these are pullbacks of a hypergeometric differential operators, and are associated to families of elliptic curves. Other known examples of 2nd order differential equations with an integral solution are associated to elliptic K3-surfaces ([5]). This includes the differential equations that came up in the proof of the transcendence of $\zeta(2)$ and $\zeta(3)$. The differential equations with integral solution we consider in this paper have 5 singularities, and are not the pullback of a hypergeometric differential equation.

Our differential equations also come from geometry, though they are of a different nature. Namely, they are the uniformizing differential equations of Teichmüller curves in genus 2. These curves were discovered by Calta and by McMullen and intensely studied from a complex-analytic point of view. This paper starts to explore arithmetic aspects of Teichmüller curves. For an introduction to Teichmüller curves, we refer to $\S$ 1. We restrict to Teichmüller curves parameterizing curves of genus 2. Let $C$ be such a Teichmüller curve.

2000 Mathematics Subject Classification. Primary 14H25; Secondary 32G15, $12 \mathrm{H} 25$.

Key words and phrases. Globally nilpotent differential operators, Teichmüller curves, S-integral solutions. 
This paper contains results in two direction. Firstly, we prove new results on Teichmüller curves in genus 2. Secondly, we show that the uniformizing differential equation of these Teichmüller curves have interesting arithmetic properties.

A new ingredient we use for studying Teichmüller curves in genus 2 is the construction of genus-2 fibrations as double coverings of ruled surfaces (following [10]). This allows us, for example, to compute the Lyapunov exponents of $C(\S 2$ ) for all Teichmüller curves $C$ in genus two. This extends a result of Bainbridge ([3]).

Teichmüller curves in genus 2 whose generating translation surface $(X, \omega)$ has a double zero are classified by McMullen ([21, 23]). These Teichmüller curves are characterized by two invariants: the discriminant $D \in \mathbb{N}$ and, if $D \equiv 1(\bmod 8)$, the spin invariant $\varepsilon \in \mathbb{Z} / 2 \mathbb{Z}$ which is the signature of a quadratic form on a certain subspace of $H^{1}(X, \mathbb{Z} / 2 \mathbb{Z})$ (§ 1). We denote by $W_{D}^{\varepsilon}$ the Teichmüller curve with discriminant $D$ and spin invariant $\varepsilon$. Surprisingly, the field of moduli of $W_{D}^{\varepsilon}$ depends on whether $D$ is a square or not, even though the spin invariant may be defined the same way in both cases.

Theorem 3.3. If $D \equiv 1(\bmod 8)$ is not a square, the field of moduli of the Teichmüller curves $W_{D}^{0}$ and $W_{D}^{1}$ is $\mathbb{Q}(\sqrt{D})$. Otherwise, the field of moduli of $W_{D}^{\varepsilon}$ is $\mathbb{Q}$.

Theorem 3.3 allows to significantly simplify Bainbridge's calculation of the orbifold Euler characteristic of $W_{D}^{\varepsilon}([3])$. It is still an open problem to determine what the $W_{D}^{\varepsilon}$ are, for example, as algebraic curves marked by their cusps and elliptic points. We solve this problem for $D=13$ and $D=17$ which are the first nontrivial cases. Note that the usual definitions of the Teichmüller curves, as Teichmüller geodesics and as locus of eigenforms with a double zero, are rather unsuitable for such a computation.

To determine $W_{D}^{\varepsilon}$, we explicitly compute an equation for the universal family $\mathcal{X}$ of curves of genus 2 parameterized by $W_{D}^{\varepsilon}(\S 7)$. We show that, for $D=13,17$, this family of curves is determined by its degenerations. The explicit equation for $\mathcal{X}$ allows us also to compute the uniformizing differential equations $L_{D}^{\varepsilon}$ of $W_{D}^{\varepsilon}(\S 8)$.

In $\S 9$ we show that $L_{D}^{\varepsilon}$ has an integral solution in a neighborhood of a cusp. We deduce this from the existence of an integral solution modulo $p^{n}$ for all $n$. The existence of approximate solutions follows from Katz' theorem on expansion coefficients ([16]). The proof of the integrality does not use the explicit equation for the differential equation $L_{D}^{\varepsilon}$, but only the existence of an integral model for $\mathcal{X}$. Therefore the proof applies to all Teichmüller curves of genus zero, under a mild hypotheses. (See $\S 9$ for the precise statement.)

Theorem 9.1. Let $W_{D}^{\varepsilon}$ be a Teichmüller curve of genus zero. There exists a finite set $\mathcal{S} \subset \mathbb{Z}$ of primes such that $L_{D}^{\varepsilon}$ admits a holomorphic $\mathcal{S}$-integral solution.

The differential operators $L_{D}^{\varepsilon}$ are counterexamples to several conjectures which fit into the circle of ideas around Grothendieck's $p$-curvature conjecture. Recall that a differential operator $L$ is globally nilpotent if the reduction of $L$ (modulo $p$ ) has nilpotent $p$-curvature for almost all $p(\S 5)$. The nilpotence of the $p$-curvature may also be characterized in terms of the existence of algebraic solutions of the reduction of $L$ modulo $p$. The conjecture of D.V. Chudnovsky and G.V. Chudnovsky (Conjecture 6.1) may be seen as an attempt to characterize the monodromy groups of globally nilpotent differential equations. A theorem of Katz ([15]) implies that $L_{D}^{\varepsilon}$ is globally nilpotent. The following result on the monodromy group of $L_{D}^{\varepsilon}$ yields counterexamples to this conjecture. 
Theorem 6.2. There exist infinitely many D such that:

(a) $\Gamma_{D}^{\varepsilon}$ is nonarithmetic,

(b) $\Gamma_{D}^{\varepsilon}$ is not commensurable to a triangle group,

(c) the uniformizing differential equation $L_{D}^{\varepsilon}$ of $\Gamma_{D}^{\varepsilon}$ may be defined over $\mathbb{Q}(\sqrt{D})$, and is globally nilpotent.

We note that (a) and (b) of Theorem 6.2 are due to McMullen ([21]). Our contribution to this theorem is linking the Teichmüller curves to the theory of differential equations. There is a finite list of discriminants $D$ such that $L_{D}^{\varepsilon}$ lives on a curve of genus zero. In this case the differential equation $L_{D}^{\varepsilon}$ does not have an algebraic solution (Proposition 6.4). This yields nonarithmetic counterexamples to a conjecture of Dwork (Conjecture 6.3).

An arithmetic counterexample to Dwork's conjecture has previously been found by Krammer ([18]). Krammer's example is the uniformizing differential equation of a Shimura curve $C=\mathbb{H} / \Gamma$. Krammer's strategy in computing the uniformizing differential equation of $\Gamma$ is to exploit the existence of a correspondence $C^{\prime} \rightrightarrows C$. A similar strategy has been used by Elkies ([13]). Krammer's strategy is unlikely to work for a nonarithmetic Fuchsian group (Remark 6.5). The reason why it is hard to find counterexamples to the conjecture of Chudnovsky-Chudnovsky appears to be that it is difficult to find globally nilpotent differential equations whose monodromy group is a nonarithmetic Fuchsian group which is not commensurable to a triangle group.

\section{Teichmüller CURVES IN Genus 2}

A Teichmüller curve is a generically injective, holomorphic map $C \rightarrow \mathcal{M}_{g}$ which is geodesic for the Teichmüller metric. Here $C$ is a smooth algebraic curve $C$ and $\mathcal{M}_{g}$ is the moduli space of curves of genus $g$. Teichmüller curves arise naturally from the study of dynamics of billiard tables. We recall the standard construction of Teichmüller curves. Let $X$ be a Riemann surface of genus $g$ and $\omega$ a holomorphic 1-form on $X$. The orbit of $(X, \omega)$ under the natural action of $\mathrm{SL}_{2}(\mathbb{R})$ on $\Omega \mathcal{T}_{g}$ projects to a geodesic disc $\mathbb{H} \rightarrow \mathcal{T}_{g} \rightarrow \mathcal{M}_{g}$ under the Teichmüller metric. If the stabilizer $\Gamma$ of $(X, \omega)$ is a lattice in $\mathrm{SL}_{2}(\mathbb{R})$, the image of the orbit in $\mathcal{M}_{g}$ is a curve. Its normalization $C=\mathbb{H} / \Gamma$ is then a Teichmüller curve. A pair $(X, \omega)$ is called a translation surface. A translation surface that generates a Teichmüller curve is called a Veech surface. The corresponding lattice $\Gamma$ is called the affine group of $C$.

More generally, one could consider Teichmüller curves generated by $(X, q)$, where $q \in$ $\Gamma\left(X,\left(\Omega_{X}^{1}\right)^{\otimes 2}\right)$ is a quadratic differential form. After replacing $C$ by a cover of degree 2 if necessary, one may assume that there exists a 1-form $\omega$ with $q=\omega^{2}$. Therefore it is no restriction to only consider Teichmüller curves $C$ which are generated by a translation surface $(X, \omega)$.

We let $\bar{C}$ be a smooth compactification of $C$ and $S:=\bar{C} \backslash C$. We may replace $C$ by a finite, unramified cover such that there exists a universal family $f: X \rightarrow C$ of smooth curves of genus $g$. Moreover, we may assume that $C=\mathbb{H} / \Gamma$ with $\Gamma$ torsion free. Then $f: X \rightarrow C$ extends to a family $\bar{f}: \bar{X} \rightarrow \bar{C}$ of stable curves $([24], \S 1.4)$.

By [24], Theorem 2.6, Teichmüller curves parameterize curves whose Jacobian has a factor of rank $r$ with real multiplication. Here $r \leq g$ is the degree of the trace field of $\Gamma$. (Recall that the trace field is the (finite) extension of $\mathbb{Q}$ which is generated by the traces of all $A \in \Gamma$.) If $(X, \omega)$ is a translation surface generating a Teichmüller curve, then $\omega$ is an eigenform for the real multiplication. 
We suppose from now on that $g=2$ which is the case we study in this paper. The de Rham cohomology of $f: \bar{X} \rightarrow \bar{C}$ decomposes as

$$
\mathcal{H}_{\mathrm{dR}}^{1}(\bar{X})=\mathcal{E}_{1} \oplus \mathcal{E}_{2}
$$

where $\mathcal{E}_{i}$ are flat vector bundles of rank 2 with logarithmic poles in $S$. We denote their Hodge filtration by $\mathcal{L}_{i} \subset \mathcal{E}_{i}$. In particular, we have

$$
f_{*} \omega_{\bar{x} / \bar{C}}=\mathcal{L}_{1} \oplus \mathcal{L}_{2}
$$

It is shown in 24], Theorem 2.6, that one of the vector bundles $\mathcal{E}_{i}$, say $\mathcal{E}_{1}$, is indigenous. This means that the Kodaira-Spencer map

$$
\Theta: \mathcal{L}_{1} \rightarrow \mathcal{E}_{1} \stackrel{\nabla}{\rightarrow} \mathcal{E}_{1} \otimes \Omega \frac{1}{C}(\log S) \rightarrow\left(\mathcal{E}_{1} / \mathcal{L}_{1}\right) \otimes \Omega_{\bar{C}}(\log S)
$$

is an isomorphism. (The local system corresponding to $\mathcal{E}_{1}$ is called maximal Higgs.) One may characterize Teichmüller curves generated by translation surfaces via the existence of an indigenous bundle $\mathcal{E}_{1}$ ([24], Theorem 5.5).

Remark 1.1. After replacing $C$ by a finite unramified cover, there exist isomorphisms $\mathcal{L}_{1} \simeq$ $\Omega \frac{1}{C}(\log S)^{1 / 2}$ and $\mathcal{E}_{1} / \mathcal{L}_{1} \simeq \Omega \frac{1}{C}(\log S)^{-1 / 2}$. In particular, $\operatorname{deg} \Omega \frac{1}{C}(\log S)=2 g(\bar{C})-2+|S|$ is even.

The rational numbers $\lambda_{1}:=1$ and $\lambda_{2}:=\operatorname{deg}\left(\mathcal{L}_{2}\right) / \operatorname{deg}\left(\mathcal{L}_{1}\right)$ are called the Lyapunov exponents of $C$. We will use the Lyapunov exponents rather then the degrees of the line bundles $\mathcal{L}_{i}$ as invariants as the Lyapunov exponents do not change if we replace $\bar{C}$ by a finite cover. We refer to [9], Proposition 8.5, for the proof that these numbers coincides with the usual definition of the Lyapunov exponents as growth rates of the Hodge norms along the Teichmüller geodesic flow.

We suppose that $(X, \omega)$ generates the Teichmüller curve $C$, via the above construction. Then $X$ is a fiber of $X$ and $\omega$ is a section of $\mathcal{L}_{1}$. There are two possibilities: $\omega$ has either two simple zeros or one double zeros. We denote by $\Omega \mathcal{M}_{2}(1,1)$ (resp. $\left.\Omega \mathcal{M}_{2}(2)\right)$ the locus of pairs $(X, \omega)$ where $\omega$ has two simple zeros (resp. one double zero).

We recall McMullen's classification of primitive Teichmüller curves in the moduli space of curves of genus $g=2$. A Teichmüller curve is primitive if it does not arise from a family of curves of lower genus via a branched covering.

There is a unique primitive Teichmüller curve corresponding to a translation surface $(X, \omega)$ for which $\omega$ has two simple zeros $([23])$. In this case, the genus of $C$ is zero and the connection on $\mathcal{E}_{1}$ has three regular singularities. Therefore the affine group of this Teichmüller curve is a triangle group. This family is a special case of the families studied in $[9]$. The curves parameterized by $C$ have real multiplication by $\mathbb{Z}[\sqrt{5}]$.

There is an infinite family of primitive Teichmüller curves corresponding to a translation surface $(X, \omega)$ for which $\omega$ has a double zero. McMullen ([22]) shows that these Teichmüller curves are characterized by two invariants: the discriminant $D$ and the spin invariant $\epsilon$. We recall the definition of these invariants.

Let $K$ be a totally real number field with $[K: \mathbb{Q}]=2$, and let $\mathcal{O}_{D} \subset K$ be an order of discriminant $D$. We denote by $W_{D} \subset \mathcal{M}_{g}$ the locus of curves of genus 2 whose Jacobian admits real multiplication by $\mathcal{O}_{D}$, and which carry an eigenform $\omega$ for the real multiplication which has a double zero at one of the 6 Weierstraß points. Then $W_{D}=\emptyset$ if $D \leq 4$. For $D \geq 5$, every irreducible component of $W_{D}$ is a Teichmüller curve. If $D \equiv 1(\bmod 8)$ and $D \neq 9$, then $W_{D}$ is the disjoint union of two curves $W_{D}^{\varepsilon}$, where $\varepsilon \in \mathbb{Z} / 2 \mathbb{Z}$ is the spin invariant. Otherwise, $W_{D}$ is irreducible. The spin invariant $\varepsilon$ may also be defined 
more conceptually as the Arf invariant of some real multiplication endomorphism acting on $\mathbb{Z} / 2 \mathbb{Z}$-cohomology $([22, \S 5)$.

To avoid a case distinction, we denote all primitive Teichmüller curves by $W_{D}^{\varepsilon}$ even if $W_{D}$ is irreducible. The curve $W_{D}^{\varepsilon}$ may be defined over a number field. (See Theorem 3.3 for a precise statement.)

\section{Computation of the Lyapunov exponents}

We let $f: \bar{X} \rightarrow \bar{C}$ be the universal family over a (finite cover of a) Teichmüller curve, as in $\S 1$. Let $(X, \omega)$ be a translation surface generating this Teichmüller curve. In this section, we compute the Lyapunov exponents $\lambda_{i}$ of $C$ by using a result of Catanese and Pignatelli ([10]) on the structure of genus-2 fibrations. This gives a new, shorter proof of a result of Bainbridge ([3]) in the case that $(X, \omega) \in \Omega \mathcal{N}_{2}(2)$. In the case that $(X, \omega) \in \Omega \mathcal{M}_{2}(1,1)$ and $C$ is imprimitive the result is new. The affine group of the (unique) primitive Teichmüller curve corresponding to a translation surface in $\Omega \mathcal{M}_{2}(1,1)$ is a triangle group. Its Lyapunov exponents were calculated in [9].

Lemma 2.1. Let $c \in \bar{C}$ be a point such that the fiber $X=\bar{X}_{c}$ is singular.

(a) The curve $X$ does not contain a separating node. In particular, $X$ does not consist of two elliptic curves meeting in one point.

(b) If $\bar{C}$ is a primitive Teichmüller curve generated by a Veech surface in $\Omega \mathcal{M}_{2}(2)$ then $X$ consists of a projective line which intersects itself in two points.

Proof. This lemma follows from some well-known results on the geometry of translation surfaces $(X, \omega)$ which we quickly recall. For a given direction in $v \in \mathbb{R}^{2}$ one considers the geodesics for $\omega$ with slope $v$. A direction is called periodic, if all geodesic are closed or join zeros of $\omega$. The translation surface decomposes into cylinders in the direction of a periodic direction, as, for example, in the horizontal direction in Figure 1. A geodesic in the interior of a cylinder is called a core curve.

The singular fibers of $\bar{f}: \bar{x} \rightarrow \bar{C}$ correspond bijectively to periodic directions, up to the action of the affine group $\Gamma$. Topologically, singular fibers of $\bar{f}: \bar{X} \rightarrow \bar{C}$ are obtained by squeezing the core curves of the cylinder decomposition of the corresponding flat surface in some direction. This is justified in [20, where flat metrics are related to hyperbolic metrics.

Suppose that a core curve of a cylinder $Z$ separates $(X, \omega)$ into two planar polygons $P_{1}$ and $P_{2}$. This implies that all sides of $P_{1}$, except for the boundary of $Z$, come in pairs that are glued by translations preserving the global orientation of the plane. Fix an orientation of the boundary of $P_{1}$. Sides glued together consequently have opposite orientation on the boundary. We have obtained a contradiction since the translation vectors of all sides of $P_{1}$ have to add up to zero.

The second statement follows from [25], Theorem 2.1 and Corollary 2.2.

The relative canonical map of a family $\bar{f}: \bar{X} \rightarrow \bar{C}$ of genus-two curves defines a rational $\operatorname{map} \varphi: \bar{x}-->\mathbb{P}:=\mathbb{P}\left(V_{1}\right)$, where $V_{1}=f_{*} \omega_{x / C}$. It is known since the work of Horikawa how to reconstruct $\bar{f}$ from the ruled surface $\mathbb{P}$ and covering data. We follow the recent nice account in [10].

By Lemma 2.1 the cokernel

$$
\tau=\operatorname{coker}\left(S^{2}\left(f_{*} \omega_{X / C}\right) \rightarrow f_{*} \omega_{X / C}^{2}\right)
$$


is zero and for the same reason $\varphi$ is actually a morphism. In this situation, the relative canonical ring of the fibration is uniquely determined ([10], page 1014 and Proposition 4.8) by a morphism

$$
\delta: \operatorname{det}\left(V_{1}\right)^{2} \rightarrow S^{6}\left(V_{1}\right) .
$$

Since in our situation $V_{1}=\mathcal{L}_{1} \oplus \mathcal{L}_{2}$ splits into eigenspaces of real multiplication, $\delta$ is a direct sum of maps between line bundles. The structure of $\delta$ is described in the following Propositions 2.2 and 2.3 .

Proposition 2.2. Let $X \rightarrow C$ be the universal family over a Teichmüller curve generated by a translation surface in $\Omega \mathcal{M}_{2}(2)$. Then we have a decomposition

$$
\delta=\oplus_{k=0}^{6} \delta(k): \mathcal{L}_{1}^{2} \otimes \mathcal{L}_{2}^{2} \rightarrow \oplus_{k}\left(\mathcal{L}_{1}^{6-k} \otimes \mathcal{L}_{2}^{k}\right)
$$

with the properties

(a) the map $\delta(0)$ is identically zero, and

(b) the map $\delta(1)$ is an isomorphism.

Proof. The decomposition of $\delta$ follows immediately from the decomposition of $f_{*} \omega_{\bar{x}} / \bar{C}$.

Fix a point $p \in \bar{C}$ and let $t$ a local parameter at $p$. Choose local sections $s_{i}$ of $\mathcal{L}_{i}$ in a neighborhood of $p$, and write

$$
\delta(k)\left(s_{1}^{\otimes 2} s_{2}^{\otimes 2}\right)=c_{k}(t) s_{1}^{\otimes 6-k} s_{2}^{\otimes(k)}
$$

for functions $c_{k},(k=0, \ldots, 6)$. The choice of local coordinates $c_{k}$ is equivalent to representing $\bar{f}$ in a neighborhood of $p$ in terms of the inhomogeneous coordinate $x=s_{1} / s_{2}$ as

$$
y^{2}=\sum_{k=0}^{6} c_{k} x^{k}
$$

Compare to [10], $\S 4$.

Recall from $\S 1$ that there exists a section $\omega_{1}$ of $\mathcal{L}_{1}$ which has a double zero. The choice of the coordinate $x$ implies that $\omega_{1}$ has a double zero at $x=\infty$, hence $c_{6}=0$. This implies (a). Since (41) represents a family of curves of genus 2 , we conclude that $c_{5} \neq 0$. This implies (b).

A similar proof yields the analogous result in the case of Teichmüller curves generated by a translation surface in $\Omega \mathcal{M}_{2}(1,1)$.

Proposition 2.3. Let $X \rightarrow C$ be the universal family over a Teichmüller curve generated by a translation surface in $\Omega M_{2}(1,1)$. Then we have a decomposition

$$
\delta=\oplus_{k=0}^{6} \delta(k): \mathcal{L}_{1}^{2} \otimes \mathcal{L}_{2}^{2} \rightarrow \oplus_{k}\left(\mathcal{L}_{1}^{6-k} \otimes \mathcal{L}_{2}^{k}\right),
$$

and $\delta(0)$ is an isomorphism.

We now use Propositions 2.2 and 2.3 to calculate the Lyapunov exponents for Teichmüller curves in genus two.

Corollary 2.4. Let $C$ be a Teichmüller curve in genus $g=2$ generated by the translation surface $(X, \omega)$. The Lyapunov exponents are

$$
\left(\lambda_{1}, \lambda_{2}\right)= \begin{cases}\{1,1 / 3\} & \text { if }(X, \omega) \in \Omega M_{2}(2), \\ \{1,1 / 2\} & \text { if }(X, \omega) \in \Omega M_{2}(1,1) .\end{cases}
$$


Proof. By definition, we have that $\lambda_{1}=1$ and $\lambda_{2}=\operatorname{deg}\left(\mathcal{L}_{2}\right) / \operatorname{deg}\left(\mathcal{L}_{1}\right)$. In the situation of Proposition 2.2 we have that

$$
2 \operatorname{deg}\left(\mathcal{L}_{1}\right)+2 \operatorname{deg}\left(\mathcal{L}_{2}\right)=\operatorname{deg}\left(\mathcal{L}_{1}\right)+5 \operatorname{deg}\left(\mathcal{L}_{2}\right) .
$$

In the situation of Proposition 2.3, we have that

$$
2 \operatorname{deg}\left(\mathcal{L}_{1}\right)+2 \operatorname{deg}\left(\mathcal{L}_{2}\right)=6 \operatorname{deg} \mathcal{L}_{2} .
$$

This implies the statement.

\section{Prototypes for singular fibers and the Galois action on the set of COMPONENTS OF $W_{D}$}

In this section, we consider primitive Teichmüller curves corresponding to a translation surface $(X, \omega) \in \Omega \mathcal{M}_{2}(2)$. Let $W_{D}$ be as in $\$ 1$. Recall that $W_{D}$ is the disjoint union of two Teichmüller curves $W_{D}^{\varepsilon}$ if $D \equiv 1(\bmod 8)$ and is a Teichmüller curve otherwise. The goal of this section is to show that if $D \equiv 1(\bmod 8)$ is not a square the field of moduli $W_{D}^{\varepsilon} \rightarrow \mathcal{M}_{2}$ is not $\mathbb{Q}$. A key ingredient is a normal form for the degenerate fibers of the corresponding universal family $\bar{f}: \bar{X}^{\varepsilon}(D) \rightarrow \bar{C}(D)$. We usually drop $D$ and $\varepsilon$ from the notation, if they are clear from the context.

McMullen ([22]) shows that the cusps of $W_{D}$ correspond to so-called splitting prototypes. We recall the definition.

Definition 3.1. A quadruple of integers $(a, b, c, e)$ is a splitting prototype of discriminant $D$ if

$$
\begin{aligned}
& D=e^{2}+4 b c, \quad 0 \leq a<\operatorname{gcd}(b, c), \quad 0<b \\
& 0<c, \quad c+e<b \quad \text { and } \quad \operatorname{gcd}(a, b, c, e)=1
\end{aligned}
$$

To a splitting prototype $(a, b, c, e)$ of discriminant $D$, we may associate a translation surface as in Figure 1, where vertical sides are glued by horizontal translations and where $\lambda=(e+\sqrt{D}) / 2$.

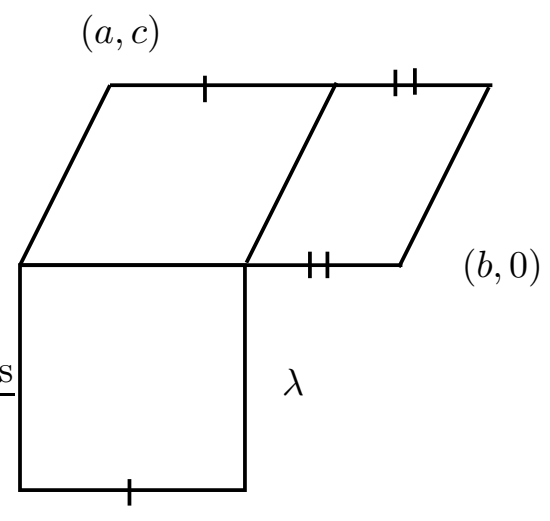

$\lambda$

Figure 1. A translation surface attached to a splitting prototype 
Let $(a, b, c, e)$ be a splitting prototype of discriminant $D$. Write $D=E F^{2}$ with $E$ square free. Then it follows from [22], Proposition 5.3, that the corresponding translation surface has spin invariant

$$
\varepsilon=\frac{e-F}{2}+(c+1)(a+b+a b) \quad(\bmod 2) .
$$

Proposition 3.2. $\quad$ (a) The singular fiber corresponding to $(a, b, c, e)$ may be given by

$$
y^{2}=(x-\mu)\left(x-\mu-b^{2}\right)^{2}\left(x-\mu-\lambda^{2}\right)^{2} \quad \text { where } \quad \mu=\frac{\lambda\left(\lambda \sigma(\lambda)-b^{2}\right)}{\sigma(\lambda)-\lambda} .
$$

Here $\sigma$ denotes the generator of $\operatorname{Gal}(\mathbb{Q}(\sqrt{D}), \mathbb{Q})$.

(b) We may, furthermore, suppose that $\omega_{1}=\mathrm{d} x / y$ and $\omega_{2}=x \mathrm{~d} x / y$ are eigenforms for real multiplication by $\mathcal{O}_{D}$.

(c) The variable $x$ is uniquely determined by (a) and (b), up to multiplication by a constant.

Proof. Lemma 2.1.(b) implies that a singular fiber $X$ of $\bar{X}$ is a projective line $\mathbb{P}_{z}^{1}$ with two pairs of points identified. We normalize the hyperelliptic involution ad hoc by $z \mapsto-z$. The Weierstraß points on the generic fiber of $\bar{X}$ specialize to the points $0, \infty$ and the two pairs of points $p,-p$ and $q,-q$ that are glued together.

Let $\omega_{1}$ be a section of $\mathcal{L}_{1}$ restricted to the singular fiber $X_{c}$. We claim that we can read off the residues of $\omega_{1}$ from the geometry of Figure 1, up to a common scalar multiple coming from replacing $\omega_{1}$ by a scalar multiple and up to \pm 1 stemming from the choice of a branch at the singularity. Recall that the singular fiber is obtained topologically by squeezing the horizontal core curves of the cylinders. Metrically, $\operatorname{diag}\left(e^{t}, e^{-t}\right)$ tends to $x_{c}$ for $\left.t \rightarrow \infty(20]\right)$. Consequently, up to indeterminacies mentioned in the claim, the residues of $\omega_{1}$ are the lengths of the core curves of the horizontal cylinders in Figure 1 . We choose one of the two possibilities for the sign, and find that

$$
\omega_{1}=\left(\frac{\lambda}{z-p}+\frac{-\lambda}{z+p}+\frac{-b}{z-q}+\frac{b}{z+q}\right) \mathrm{d} z .
$$

By assumption, $\omega_{1}$ has a double zero, which is either at $x=0$ or at $x=\infty$. We may assume that $\omega_{1}$ has a double zero at $x=\infty$. This implies that $\lambda p=b q$. After multiplying $z$ by a suitable constant, we may assume that $q=\lambda$ and $p=b$.

Since $b \in \mathbb{Q}$, it follows that $b$ is fixed by $\sigma$. Therefore

$$
\omega_{2}=\left(\frac{\sigma(\lambda)}{z-p}+\frac{\sigma(-\lambda)}{z+p}+\frac{-b}{z-q}+\frac{b}{z+q}\right) \mathrm{d} z .
$$

Note that $\omega_{2}$ has zeros in $z= \pm \sqrt{\mu}$, for some $\mu$.

In terms of the coordinates $x^{\prime}=z^{2}$ and $y=z\left(x^{\prime}-\lambda^{2}\right)\left(x^{\prime}-b^{2}\right)$, we find that, up to multiplicative constants, $\omega_{1}=\mathrm{d} x / y$ and $\omega_{2}=\left(x^{\prime}-\mu\right) \mathrm{d} x^{\prime} / y$. Therefore defining $x=x^{\prime}+\mu$ yields (b). The coordinates $x$ and $y$ obviously satisfy the relation in (a). The uniqueness assertion (c) follows immediately from our normalization.

Theorem 3.3. (a) Suppose that $D=d^{2} \equiv 1(\bmod 8)$. Then the components $W_{D}^{\varepsilon}$ of $W_{D}$ are fixed by the absolute Galois group $G_{\mathbb{Q}}$, i.e. $G_{\mathbb{Q}}$ acts trivially on the map $W_{D}^{\varepsilon} \rightarrow \mathcal{M}_{2}$.

(b) If $D \equiv 1 \bmod 8$ is not a square, the action of $\operatorname{Gal}(\mathbb{Q}(\sqrt{D}) / \mathbb{Q})$ sends $W_{D}^{0} \rightarrow \mathcal{M}_{2}$ to $W_{D}^{1} \rightarrow \mathcal{M}_{2}$. In particular, the field of moduli of $W_{D}^{\varepsilon} \rightarrow \mathcal{M}_{2}$ is not $\mathbb{Q}$. 
This theorem may be reformulated as follows. The moduli map $W_{D}^{\varepsilon} \rightarrow \mathcal{M}_{2}$ may be defined over $\mathbb{Q}$ if and only if $W_{D}^{\varepsilon}$ is imprimitive or $W_{D}$ is irreducible. An immediate consequence is that orbifold the Euler characteristics of $W_{D}^{0}$ and $W_{D}^{1}$ coincide. The proof in [3] needed a careful analysis of intersection numbers to arrive at this conclusion.

Proof. Suppose that $D \equiv 1(\bmod 8)$ is a square. Then $W_{D}^{\varepsilon}$ is imprimitive, and the theorem follows from ([22], Theorem 6.1).

Suppose that $D \equiv 1(\bmod 8)$ is not a square. For the second statement, recall from [24], Corollary 5.4, that $G_{\mathbb{Q}}$ acts on the set of Teichmüller curves. Moreover, given a family of curves over a Teichmüller curve, $G_{\mathbb{Q}}$ acts on the family of Jacobians, and maps eigenforms for real multiplication to eigenforms. Obviously, $G_{\mathbb{Q}}$ also preserves the multiplicity of the zeros of both eigenforms.

By the classification of Teichmüller curves in $\Omega \mathcal{N}_{2}(2)$, the absolute Galois group $G_{\mathbb{Q}}$ acts either trivially or via a group of order 2 . Let $c \in \bar{C}$ be a cusp. Then to $c$ we associate a triple $\left(X, \omega_{1}, \omega_{2}\right)$, where $X$ is the stable curve $\bar{X}_{c}$ and $\omega_{i}$ are holomorphic sections of $\left.\varepsilon_{i}\right|_{c}$ which are eigenforms for real multiplication.

The boundary divisors of $\overline{\mathcal{M}}_{2}$ are defined over $\mathbb{Q}$. To show that the $G_{\mathbb{Q}}$-action is nontrivial, it suffices therefore to find a cusp $c_{0}$ of $W_{D}^{0}$ whose Galois conjugate is a cusp $c_{1}$ of $W_{D}^{1}$ and such that the triples $\left(X_{j}, \omega_{1, j}, \omega_{2, j}\right)$ are not isomorphic.

We consider the splitting prototypes $(0,(D-1) / 4,1,1)$ and $(0,(D-1) / 4,1,-1)$ which have different spin invariant by (5). We write $\lambda_{e}=(e+\sqrt{D}) / 2$ for $e= \pm 1$. Let $\mu_{e}$ be given as in equation (6). One easily calculates that

$$
\sigma\left(\lambda_{1}^{2}\right)=\lambda_{-1}^{2} \quad \text { and } \quad \sigma\left(\mu_{1}\right)=\mu_{-1} .
$$

Consequently, $\sigma$ applied to the equation (6) for the prototype $(0,(D-1) / 4,1,1)$ yields the corresponding equation attached to the prototype $(0,(D-1) / 4,1,-1)$. We thus have found a cusp that is moved by $G_{\mathbb{Q}}$ from $W_{D}^{0}$ to $W_{D}^{1}$.

It remains to check that the triples attached to the prototypes are not isomorphic. Equivalently, we have to check that the curves $X^{\varepsilon(e)}$ given by the normal form (6) are not isomorphic through an isomorphism fixing 0 and $\infty$. Suppose not. Then the isomorphism $X^{\varepsilon(-1)} \rightarrow X^{\varepsilon(1)}$ corresponds to the change of coordinates $x \mapsto x \mu_{1} / \mu_{-1}$. Since $\mu_{1}=$ $\sigma\left(\mu_{-1}\right) \neq \mu_{-1}$, we find that

$$
\frac{\mu_{1}}{\mu_{-1}}\left(\mu_{-1}+b^{2}\right)=\left(\mu_{1}+\lambda_{1}^{2}\right) .
$$

This is equivalent to

$$
\frac{\mu_{1}}{\mu_{-1}}=\frac{\lambda_{1}^{2}}{b^{2}}
$$

One computes that

$$
\frac{\mu_{1}}{\mu_{-1}}=-\frac{\lambda_{1}}{\lambda_{-1}}=\frac{(1+\sqrt{D})^{2}}{D-1}, \quad \text { and } \quad \frac{\lambda_{1}^{2}}{b^{2}}=\frac{4(1+\sqrt{D})^{2}}{D-1} .
$$

We obtain a contradiction.

\section{TRiangle Groups}

In this section we recall a result of McMullen which says that there are infinitely many Teichmüller curves in genus 2 whose affine group is not commensurable to a triangle group. This is a key ingredient in showing that infinitely many Teichmüller curves in genus 2 are counterexamples to the conjectures of Dwork and Chudnovsky-Chudnovsky ( $\S$ [6). 
Let $f: X \rightarrow C$ be the universal family over (a finite cover of) a primitive Teichmüller curve in genus $g=2$, as in $\$ 1$. The elliptic points are the images in $C$ of the points in the complex upper half plane $\mathbb{H}$ with nontrivial stabilizer. If $z \in \mathbb{H}$ has nontrivial stabilizer $\Gamma_{z}$, then $\Gamma_{z}$ is finite. After replacing $C$ by a finite cover, one may suppose that $\Gamma$ is torsion free. If $\Gamma$ is torsion free there are no elliptic points. This will sometimes be convenient. Recall that $K=\mathbb{Q}(\sqrt{D})$ is the trace field of $\Gamma\left(W_{D}^{\varepsilon}\right)$.

A Fuchsian group $\Gamma$ is a triangle group if $g(\bar{C})=0$ and the set $\left\{\tau_{i}\right\}$ of cusps and elliptic points has cardinality 3. A triangle group is denoted by $\Gamma=\Delta\left(n_{1}, n_{2}, n_{3}\right)$, where $n_{i}=\infty$ if $\tau_{i}$ is a cusp and $n_{i}$ is the order of the stabilizer if $\tau_{i}$ is an elliptic point. Teichmüller curves always have cusps, therefore we may assume that $n_{3}=\infty$ ([27]). The trace field of the triangle group $\Delta\left(n_{1}, n_{2}, \infty\right)$ is $\mathbb{Q}\left(\zeta_{n_{1}}+\zeta_{n_{1}}^{-1}, \zeta_{n_{2}}+\zeta_{n_{2}}^{-1}\right)$, where $\zeta_{n_{j}}$ is a primitive $n_{j}$ th root if unity of $n_{j}$ is finite and is 1 otherwise.

The following lemma is proved by McMullen $([21], \S 9)$. We recall the proof for the convenience of the reader.

Lemma 4.1. Let $D$ be the discriminant of a real quadratic field of the form $D=4 E$ or $D=E$ with $E$ square free. Let $C$ be a primitive Teichmüller curve in genus $g=2$ of discriminant $D$. Suppose that the affine group, $\Gamma$, of $C$ is commensurable to a triangle group. Then $C=W_{D}^{\varepsilon}$ with $D \leq 12$.

Proof. Let $D$ be as in the statement of the lemma. Suppose that the affine group of $W_{D}^{\varepsilon}$ is commensurable to a triangle group $\Delta(n, m, \infty)$. Commensurable Fuchsian groups have the same trace field, therefore $\mathbb{Q}(\sqrt{D})=\mathbb{Q}\left(\zeta_{n}+\zeta_{n}^{-1}, \zeta_{m}+\zeta_{m}^{-1}\right)$. This is only possible for $D \in\{5,8,12\}$.

Remark 4.2. It is an open question whether there are infinitely many primitive Teichmüller curves in genus 2 whose affine group is commensurable to a triangle group. If $D$ is the discriminant of such a Teichmüller curve, then Lemma 4.1 implies that $D=4 E F^{2}$ or $E F^{2}$ with $E$ square free and $D / F^{2} \leq 12$.

\section{Generalities on differential equations}

In this section we recall some generalities on flat vector bundles. Among other things we recall the connection between flat vector bundles and differential equations, and define global nilpotence.

Let $(\mathcal{E}, \nabla)$ be a flat vector bundle of rank 2 on a smooth projective curve $\bar{C}$ with regular singularities in $\tau_{1}, \ldots, \tau_{r} \in \bar{C}$. In the case that $\bar{C}$ is a Teichmüller curve, the set of singularities is the union of the cusps with the elliptic points. Let $t$ be a local parameter of $\bar{C}$ at $\tau_{i}$, and let $\mathfrak{m}_{\tau_{i}}$ the maximal ideal of the local ring $\mathcal{O}_{\bar{C}}, \tau_{i}$. The monodromy operator $\mu_{i}$ is the endomorphism of the fiber $\left.\mathcal{E}\right|_{\tau_{i}}=\mathcal{E}_{\tau_{i}} / \mathfrak{m}_{\tau_{i}} \cdot \mathcal{E}_{\tau_{i}}$ defined by $\nabla(t \partial / \partial t)$. One checks that $\mu_{i}$ does not depend on the choice of $t$.

Definition 5.1. The local exponents $\gamma_{1, \tau_{i}}, \gamma_{2, \tau_{i}}$ of $\mathcal{E}$ at $\tau_{i}$ are the eigenvalues of $\mu_{i}$.

If $\tau_{i}$ is a cusp then $\mu_{i}$ is quasi-unipotent. This implies that $\gamma_{1, \tau_{i}} \equiv \gamma_{2, \tau_{i}}(\bmod \mathbb{Z})$.

In the case that $g(\bar{C})=0$, a flat vector bundle $\mathcal{E}$ of rank 2 corresponds to a differential equation, as follows. Let $t$ be a parameter of $\bar{C}$. Choose a (rational) section $s$ of $\mathcal{E}$ such that $s$ and $s^{\prime}:=\partial s / \partial t$ are generically independent. Such a section is called a cyclic vector. Since the rank of $\mathcal{E}$ is 2 , there exist rational functions $p_{1}, p_{2} \in k(x)$ such that

$$
s^{\prime \prime}+p_{1} s^{\prime}+p_{2} s=0 .
$$


The differential operator $L=(\partial / \partial t)^{2}+p_{1}(\partial / \partial t)+p_{2}$ is Fuchsian. Its singularities are the singularities of $\nabla$, and possibly $t=\infty$. Recall that this means that $\operatorname{ord}_{x_{j}} p_{i} \geq-i$. The differential operator $L$ is called the differential operator associated with $(\mathcal{E}, s)$. This defines an equivalence between differential equations and vector bundles with a section.

If $\mathcal{E}$ possesses a nontrivial Hodge filtration $\mathcal{L} \subset \mathcal{E}$, for example if $\mathcal{E}$ is indigenous, we always choose $s$ to be a rational section of $\mathcal{L}$. Replacing $s$ by a multiple changes $L$ to an equivalent differential operator. If $\mathcal{E}$ is indigenous the differential operator is just the uniformizing differential equation corresponding to $\mathbb{H} \rightarrow \mathbb{H} / \Gamma=C$, as defined in [28, $\S 5.2$. This may be seen by remarking that the affine group $\Gamma$ is the monodromy group of $L$.

The notion of local exponents we defined above agrees with the classical notion of local exponents of a differential operator. Write $p_{j}=\sum_{n \geq-j} c_{j, n} t^{n}$. Then the local exponents are the roots of the indicial equation $t(t-1)+t p_{1,-1}+p_{2,-2}=0$.

If $L$ is hypergeometric, i.e. if $L$ has exactly three singularities which we may suppose to be $\{0,1, \infty\}, L$ is determined by its local exponents. This is no longer the case if $L$ has more than three singularities. Namely, apart from the position of the singularities and the local exponents, $L$ also depends on the so called accessory parameters. The main problem in determining the differential equation corresponding to $W_{D}^{\varepsilon}$ explicitly in $₫ 8$ is to determine the accessory parameters.

We now recall from [15] the notion of a globally nilpotent differential operator. Let $R$ be an integral domain which is finitely generated (as ring) over $\mathbb{Z}$, and whose fraction field $\operatorname{Frac}(R)$ has characteristic zero. Let $\pi: \overline{\mathrm{C}} \rightarrow \operatorname{Spec}(R)$ be a smooth morphism of relative dimension 1. (In our case, $R$ is an order in an number field with finitely many primes inverted, and $\overline{\mathcal{C}}$ will be a model of the Teichmüller curve $\bar{C}$ over $R$.) Let $\mathcal{D}$ be an étale divisor on $\overline{\mathcal{C}}$. We let $(\mathcal{E}, \nabla)$ be a flat vector bundle of rank 2 on $\bar{C}=\overline{\mathcal{C}} \otimes_{R} \operatorname{Frac}(R)$ with regular singularities in $D=\mathcal{D} \otimes_{R} \operatorname{Frac}(R)$. Let $p$ be a prime number which is not invertible on $\overline{\mathcal{C}}$, and let $\wp$ be a prime ideal of $R$ above $p$. Write $\mathbb{F}_{q}=R / \wp$. Reduction modulo $p$ defines a flat vector bundle $\mathcal{E}_{\wp}:=\mathcal{E} \otimes \mathbb{F}_{q}$ on $\bar{C}_{\wp}:=\overline{\mathcal{C}} \otimes \mathbb{F}_{q}$.

Define $\mathcal{T}:=\left(\Omega_{\bar{C}_{\wp} / k}(\log \mathcal{D} \otimes k)\right)^{\otimes-p}$. The p-curvature of $\mathcal{E}_{\wp}$ is an $\mathcal{O}_{\bar{C}_{\wp}}$-linear morphism $\Psi_{\mathcal{E}}: \mathcal{T} \rightarrow \operatorname{End}_{\mathcal{O}_{\bar{C}_{\wp}}}(\mathcal{E})$ defined by

$$
\Psi_{\mathcal{E}}\left(D^{\otimes p}\right):=\nabla(D)^{\otimes p}-\nabla\left(D^{\otimes p}\right) .
$$

For details, we refer to [15], $\S 5$.

Definition 5.2. (a) The $p$-curvature of $\mathcal{E}_{\wp}$ is nilpotent if $\Psi_{\mathcal{E}_{\wp}}$ consists of nilpotent endomorphisms.

(b) The flat vector bundle $\mathcal{E}$ is globally nilpotent if the $p$-curvature $\mathcal{E}_{\wp}$ is nilpotent for all but finitely many $\wp$.

(c) If $L$ is a differential operator of order 2 , we say that $L$ has nilpotent $p$-curvature if the $p$-curvature of the corresponding flat vector bundle is nilpotent.

Katz' Theorem ([15], Theorem 10.0) states that a flat vector bundle $(\mathcal{E}, \nabla)$ which is the direct factor of the relative de Rham cohomology of a family of curves defined over a number field is globally nilpotent. The local exponents of globally nilpotent flat vector bundles are rational numbers.

If $g(\bar{C})=0$, one may rephrase the notion of nilpotent $p$-curvature, as follows $([14])$. We let $\wp \mid p$ be invertible on $\overline{\mathcal{C}}$, and write $L_{\wp}$ for the differential operator corresponding to $\mathcal{E}_{\wp}$. 
Since the local exponents of $L$ are rational numbers in our situation, the $p$-curvature of $\mathcal{E}_{\wp}$ is nilpotent if and only if $L_{\wp}$ has a polynomial solution.

The $p$-curvature of $\mathcal{E}_{\wp}$ is identically zero if $\Psi_{\mathcal{E}_{\wp}}$ is zero. If $g(C)=0$, this is equivalent to saying that the corresponding differential operator $L_{\wp}$ has a basis of polynomial solutions. Since Teichmüller curves always have a cusp, it follows from [14], Proposition 5.1, that the $p$-curvature of the differential operators we consider do not have zero $p$-curvature.

The analog of this notion in characteristic zero is that $L$ has a basis of algebraic solutions. Here $u \in \mathbb{C}((t))$ is called algebraic is if it algebraic over $\mathbb{C}(t)$. The differential operator $L$ has a basis of algebraic solutions if and only if its monodromy group is finite. Since the monodromy group of the uniformizing differential equation $L$ of a Teichmüller curves is never finite, $L$ does not have a basis of algebraic solutions.

We state a well-known result on solutions of differential operators in characteristic zero. It follows immediately from Fuchs' Theorem ([6], Theorem 2.9). Note that if $\tau$ is a cusp of $L$ then after replacing $\bar{C}$ by a finite cover and $L$ by an equivalent differential operator, we may assume that the local exponents of $L$ at $\tau$ are $(0,0)$. Therefore the condition in Lemma 5.3 is no serious restriction.

Lemma 5.3. Let $L$ be a Fuchsian differential operator of order 2. Suppose that $\tau$ is a cusp, and let $t$ be a local parameter in $\tau$. Assume that the local exponents of $L$ at $\tau$ are $(0,0)$. Then there exists a unique solution $u \in \mathbb{C}[[t]]$ of $L$ around $t=0$ with the property that $u(x=0)=1$.

\section{Counterexamples to the COnjectures of Dwork And Chudnovsky-Chudnovsky}

In this section we use the results from the previous sections to show that the $W_{D}^{\varepsilon}$ 's provide counterexamples to conjectures of Chudnovsky-Chudnovsky and Dwork. The main ingredients of the proofs can already be found in [21]. Our contribution here is linking the theory of Teichmüller curves with that of differential equations. In $\S 8$ we then find an explicit formula for some of the differential equations. This gives then also explicit counterexamples to these conjectures.

The following conjecture is stated by Chudnovsky and Chudnovsky in [11. The original conjecture is stated in the language of differential operators, i.e. Chudnovsky-Chudnovsky assume that the genus of $\bar{C}$ is 0 . But there is no need for this restriction (as both variants of the conjecture are wrong).

Conjecture 6.1 (Chudnovsky-Chudnovsky). Let $\bar{C}$ be a smooth projective curve defined over a number field. Let $(\mathcal{E}, \nabla)$ be an indigenous bundle on $\bar{C}$, and let $\Gamma \subset \mathrm{PSL}_{2}(\mathbb{R})$ be the monodromy group of $\mathcal{E}$. Suppose that $\mathcal{E}$ is globally nilpotent. Then $\Gamma$ is either arithmetic or commensurable to a triangle group.

We now return to the situation that $C=\mathbb{H} / \Gamma$ is a primitive Teichmüller curve in genus 2 , of discriminant $D$. We suppose that the affine group $\Gamma$ of $C$ is not commensurable to a triangle group (Lemma 4.1). Recall that $H_{\mathrm{dR}}^{1}(X)=\mathcal{E}_{1} \oplus \mathcal{E}_{2}$ is a decomposition of flat vector bundles of rank 2 ( $(1$ ). We remark that Proposition 6.2 holds for all Teichmüller curves whose affine group is not commensurable to a triangle group. One could extend the result to the Teichmüller curves in genus $g=3,4$ which where found by McMullen. Proposition 6.2, together with Lemma 4.1, produces counterexamples to Conjecture 6.1.

Proposition 6.2. $\quad$ (a) The flat vector bundles $\mathcal{E}_{i}$ are globally nilpotent.

(b) Suppose that $D \geq 13$. Then $\Gamma$ is nonarithmetic. 
Proof. Part (a) follows from the theorem of Katz ([15], Theorem 10.0). Lemma4.1 implies that $\Gamma$ is not commensurable to a triangle group. Since $C$ is a primitive Teichmüller curve in genus $g=2$, the affine group $\Gamma$ is not arithmetic ([24], Corollary 2.10.)

Note that we do not know a priori which are the finitely many values $p$ for which the $p$-curvature of $\mathcal{E}_{i}$ is not nilpotent. For the families of curves corresponding to $W_{13}$ and $W_{17}^{\varepsilon}$ we answer this question in Proposition 10.1.

In the literature one also finds variants of Conjecture 6.1, omitting either the condition that $\bar{C}$ is defined over a number field or that $\mathcal{E}$ is globally nilpotent. Proposition 6.2 shows that these variants also do not hold.

We now turn to Dwork's Conjecture ([12], Conjecture 7.4).

Conjecture 6.3 (Dwork). Let $\bar{C}$ be a smooth projective curve of genus 0 defined over a number field, and let $(\mathcal{E}, \nabla)$ be a flat vector bundle of rank 2 which is globally nilpotent. Then either the monodromy group of $\mathcal{E}$ is commensurable to a triangle group or $\mathcal{E}$ has an algebraic solution.

Proposition 6.4. Let $C$ be a primitive Teichmüller curve in genus 2 of discriminant $D \geq 13$. Suppose that $g(C)=0$. Then the flat vector bundle $\mathcal{E}_{1}$ does not admit an algebraic solution.

Proof. Let $L_{1}$ be the differential operator corresponding to $\mathcal{E}_{1}$. Let $\left\{\tau_{1}, \ldots, \tau_{r}\right\}$ be the set of singularities, and let $\xi \in \bar{C} \backslash\left\{\tau_{i}\right\}$ be a base point. Analytic continuation defines the monodromy representation

$$
\rho: \pi_{1}\left(\bar{C} \backslash\left\{\tau_{i}\right\}, \xi\right) \rightarrow \mathrm{PGL}_{2}(\mathbb{C}),
$$

which has image $\Gamma$. Since $\Gamma$ is the affine group of a Teichmüller curve, it has at least one cusp. In particular, $\Gamma$ is not finite. This implies that $\Gamma$ does not have a basis of algebraic solutions.

Suppose that $L_{1}$ has an algebraic solution $u$. For every loop $\gamma$ in $\pi_{1}\left(\bar{C} \backslash\left\{\tau_{i}\right\}, \xi\right)$, the image of $u$ under the monodromy matrix $\rho(\gamma)$ is again an algebraic solution of $L_{1}$. Since $L_{1}$ does not have a basis of solutions, this solution is again $u$ (up to a nonzero constant). This implies that $u$ is in fact a rational function. Since the genus of $C$ is zero, we find a contradiction.

McMullen ([21], Theorem 9.8) shows that the Teichmüller curves $W_{D}^{\varepsilon}$ for discriminant $D \in\{5,8,12,13,17,21,28,29,33\}$ are rational. Therefore Proposition 6.4 is a nonempty statement. In particular, together with Lemma 4.1, it also follows that there exist differential equations whose monodromy group is nonarithmetic which are counterexamples to Dwork's Conjecture.

Remark 6.5. An arithmetic counterexample to Dwork's conjecture has previously been found by Krammer ([18]). Krammer considers an arithmetic Fuchsian group $\Gamma \subset \mathrm{SL}_{2}(\mathbb{R})$ which is not commensurable to a triangle group such that $C=\mathbb{H} / \Gamma$ is a Shimura curve. To compute the uniformizing differential equation $L$, Krammer finds a subgroup of finite index of $\Gamma$ of the form $\Gamma^{\prime}:=\Gamma \cap g \Gamma g^{-1}$, where $g$ is an element of the commensurator of $\Gamma$. This yields a correspondence $\mathbb{H} / \Gamma^{\prime} \rightrightarrows C$ which allows him to determine $L$. A similar method for computing the uniformizing differential equation of a Shimura curve has also been used by Elkies ([13]).

In Krammer's approach it is essential to consider an arithmetic lattice $\Gamma$. Namely, for nonarithmetic lattices $\Gamma$ the subgroup $\Gamma \cap g \Gamma g^{-1}$ is unlikely to have finite index, since in 
this case the commensurator has finite index in $\Gamma$ ([19], Theorem B). The reason why it is hard to find counterexamples to the conjecture of Chudnovsky-Chudnovsky appears to be that it is difficult to find differential equations which correspond to a nonarithmetic groups which is not a triangle group.

\section{An equation for the Teichmüller curves $W_{13}$ And $W_{17}^{\varepsilon}$}

In the section we explicitly calculate an equation for the family of curves corresponding to the Teichmüller curves $W_{13}$ and $W_{17}^{\varepsilon}$. We give the proof only for $D=17$, and leave the case $D=13$ to the reader. We work over a double cover $\bar{C} \rightarrow \bar{W}_{17}^{\varepsilon}$. We start by assuming that there exists a universal family $f: \bar{X} \rightarrow \bar{C}$. In Lemma 7.1, we derive necessary conditions for the family $\bar{X}$. We then show that there is a (in fact unique) family of curves satisfying these conditions. We deduce that this family is the universal family corresponding to the Teichmüller $W_{17}^{\varepsilon}$.

Using the algorithm of [21], Theorem 9.8, one finds that the Teichmüller curve $\bar{W}_{17}^{\varepsilon}$ is a projective line with 3 cusps and one elliptic point of order two. We may choose a parameter $s$ of $\bar{W}_{17}^{\varepsilon}$ such that the elliptic point is $s=-1$ and such that $s=1, \infty$ are cusps. We consider a cover $\pi: \bar{C} \rightarrow \bar{W}_{17}^{\varepsilon}$ of degree 2 which is branched at $s=-1, \infty$. Then $\bar{C}$ is also a projective line; we may choose a coordinate $t$ of $\bar{C}$ such that $s=\left(t^{2}+1\right) / 2 t$. It follows that the set of cusps on $\bar{C}$ is $S=\{0,1, \infty, \tau, 1 / \tau\}$, for some $\tau \in \mathbb{R}$ (in fact, we will see that $\tau \in \mathbb{Q}(\sqrt{17})$ ).

Suppose there exists a family $\bar{f}: \bar{X} \rightarrow \bar{C}$ of stable curves of genus 2 such that the moduli map $\bar{C} \rightarrow \overline{\mathcal{M}}_{2}$ factors through $\bar{W}_{17}^{\varepsilon}$. Since $\operatorname{deg}\left(\Omega \frac{1}{C}(\log S)\right)=|S|-2=3$ is odd, we need to replace $\bar{C}$ by a cover of degree 2 the define the bundles $\mathcal{L}_{i}$ (Remark 1.1). Let $\tilde{C} \rightarrow \bar{C}$ be the cover of degree 2 which is branched at $t=0, \infty$, and let $\tilde{S} \subset \tilde{C}$ be the inverse image of $S$. On $\tilde{C}$ we have that $2 \operatorname{deg}\left(\tilde{\mathcal{L}}_{1}\right)=-2 \operatorname{deg}\left(\tilde{\mathcal{E}}_{1} / \tilde{\mathcal{L}}_{1}\right)=\operatorname{deg}\left(\Omega_{\tilde{C}}^{1}(\log \tilde{S})\right)=|\tilde{S}|-2=6$. We conclude that $\operatorname{deg}\left(\tilde{\mathcal{L}}_{1}\right)=3$ and $\operatorname{deg}\left(\tilde{\mathcal{L}}_{2}\right)=\operatorname{deg}\left(\tilde{\mathcal{L}}_{1}\right) / 3=1$ (Corollary 2.4). Since in the sequel only $\mathcal{L}_{1}^{i} \otimes \mathcal{L}_{2}^{j}$ with $i+j$ even appear, it suffices to work on $\bar{C}$.

We use the notation of $\S 2$. In particular, we let $\delta(k): \mathcal{L}_{1} \otimes \mathcal{L}_{2} \rightarrow \mathcal{L}_{1}^{k} \otimes \mathcal{L}_{2}^{6-k}$ be the maps defined in Proposition 2.2. Let $s_{i}$ be a holomorphic section of $\mathcal{L}_{i}$ which only has zeros in $t=0$. (These sections are unique up to a $\mathbb{C}^{\times}$-multiple.) As in Proposition 2.2. we obtain functions $c_{k}$ such that $\bar{X}$ is given by the equation

$$
y^{2}=g_{17}(x):=\sum_{k=0}^{5} c_{k} x^{i}, \quad \text { where } x=s_{1} / s_{2} .
$$

The calculation of $\operatorname{deg} \tilde{\mathcal{L}}_{i}$ implies that $\operatorname{deg}_{t}\left(c_{k}\right)=5-k$.

The following lemma follows immediately from the classification of the cusps $(\S[3)$.

Lemma 7.1. Suppose that there exists a family of stable curves $\bar{f}: \bar{X} \rightarrow \bar{C}$ of genus 2 such that the moduli map $\bar{C} \rightarrow \overline{\mathcal{M}}_{2}$ factors through $\bar{W}_{D}^{\varepsilon}$.

We may choose a coordinate $t$ on $\bar{C} \backslash \infty$ such that, if $s_{i}$ is the unique holomorphic section of $\mathcal{L}_{i}$ up to $\mathbb{C}^{*}$ with zeros concentrated at $t=0$, then $c_{6-k}$ as in equation (4) is a polynomial of degree $k-1$ and satisfies

$$
c_{k}(1 / t)=t^{5-k} c_{k}(t)
$$

Then the following holds:

(a) the fiber over $t=0$ has an equation (6) (up to rescaling $x$ to $\rho_{0} x$ ) for the splitting prototype $(0,4,1,1)$ if $\varepsilon=1$ and $(0,4,1,-1)$ if $\varepsilon=0$, 
(b) the fiber over $t=1$ has an equation (6) (up to rescaling $x$ to $\rho_{1} x$ ) for the splitting prototype $(1,2,2,-1)$ if $\varepsilon=1$ and $(0,2,2,-1)$ if $\varepsilon=0$,

(c) there is a $\lambda \in \mathbb{R}$ such that the fiber over $t=\lambda$ has an equation (6) (up to rescaling $x$ to $\left.\rho_{\lambda} x\right)$ for the splitting prototype $(0,2,1,-3)$ if $\varepsilon=1$ and $(0,1,2,-3)$ if $\varepsilon=0$.

We first consider the case that $\varepsilon=1$. Define

$$
\begin{aligned}
c_{0}=( & 425765 \sqrt{17}+1755475) t^{5}+(-5289173-1282803 \sqrt{17}) t^{4}+ \\
& +(857038 \sqrt{17}+3533762) t^{3}+(857038 \sqrt{17}+3533762) t^{2}+ \\
& +(-5289173-1282803 \sqrt{17}) t+425765 \sqrt{17}+1755475 \\
c_{1}=\frac{1}{2} & \left((80325 \sqrt{17}+331187) t^{4}+(-310964 \sqrt{17}-1281964) t^{3}+\right. \\
& +(461278 \sqrt{17}+1901714) t^{2}+(-310964 \sqrt{17}-1281964) t+ \\
& +80325 \sqrt{17}+331187), \\
c_{2}=\frac{1}{4} & \left((-3825 \sqrt{17}-15783) t^{3}+(3825 \sqrt{17}+15687) t^{2}+(3825 \sqrt{17}+15687) t+\right. \\
& +3825 \sqrt{17}-15783), \\
c_{3}=\frac{1}{8} & \left((-1105 \sqrt{17}-4551) t^{2}+(2210 \sqrt{17}+8918) t-1105 \sqrt{17}-4551\right), \\
c_{4}=3 & +3 t, \quad c_{5}=1 .
\end{aligned}
$$

The following proposition follows by straightforward verification.

Proposition 7.2. The family of stable curves $\bar{f}: \bar{x} \rightarrow \bar{C}$ defined by (8, 9) is the unique family satisfying the conditions of Lemma 7.1.

The curve for $t=-1$ is isomorphic to

$$
y^{2}=x\left(x^{2}-1\right)(x-a)(x-1 / a), \quad \text { where } \quad a=20+5 \sqrt{17}+2 \sqrt{206+50 \sqrt{17}},
$$

which can been calculated by Silhol's algorithm ([26]).

One may deduce from the uniqueness in Proposition 7.2 that (8) is the universal family of curves corresponding to $W_{17}^{\varepsilon}$. In $\S \mathbb{8}$, we follow an alternative approach to prove this. Namely, we show the existence of an indigenous bundle on $\bar{C}$.

It suffices to consider $\varepsilon=1$. One obtains an equation for the universal family corresponding to $W_{17}^{0}$ by replacing $\sqrt{17}$ by $-\sqrt{17}$ (Theorem 3.3 ).

We now define a family of curves which will turn out to be the family of curves corresponding to $W_{13}$. One may formulate the analog of Lemma 7.1 also in this case, and show that the family of curves below satisfies these necessary conditions.

Using the algorithm in [21], Theorem 9.8, one finds that the Teichmüller curve $\bar{W}_{13}$ is a projective curve with three cusps and an elliptic point of order 2 . As in the case that $D=17$, we let $\bar{C}_{13} \rightarrow \bar{W}_{13}$ be a cover which branches at the elliptic point and one of the cusps. On $\bar{C}_{13}$ we then have a set, $S_{13}$, of 5 cusps. We may choose a parameter $t$ of $\bar{C}_{13}$ such that $S_{13}=\left\{0,1, \infty, \rho, \rho^{-1}\right\}$. We consider a family $\bar{X}_{13}$ of stable curve of genus 2 defined by

$$
y^{2}=g_{13}(x)=\sum_{k=0}^{5} c_{k} x^{k}
$$


where

(11)

$$
\begin{aligned}
c_{0}= & \frac{1}{2^{15}}\left((-1585778688 \sqrt{13}+5717606400) t^{5}+(4758908544 \sqrt{13}-17158488768) t^{4}+\right. \\
& +(11440882287-3173129856 \sqrt{13}) t^{3}+(11440882287-3173129856 \sqrt{13}) t^{2}+ \\
& +(4758908544 \sqrt{13}-17158488768) t-1585778688 \sqrt{13}+5717606400), \\
c_{1}= & \frac{1}{2^{12}}\left((-31629312 \sqrt{13}+114041088) t^{4}+(124405632 \sqrt{13}-448550784) t^{3}+\right. \\
& +(-185552640 \sqrt{13}+669019797) t^{2}+(124405632 \sqrt{13}-448550784) t+ \\
& \quad-31629312 \sqrt{13}+114041088), \\
c_{2}= & \frac{1}{2^{5}}\left((7488 \sqrt{13}-27000) t^{3}+(-7488 \sqrt{13}+26991) t^{2}+(-7488 \sqrt{13}+26991) t+\right. \\
& \quad+7488 \sqrt{13}-27000), \\
c_{3}= & \frac{1}{2^{6}}\left((-14992+4160 \sqrt{13}) t^{2}+(30011-8320 \sqrt{13}) t-14992+4160 \sqrt{13}\right) \\
c_{4}= & t+1, \quad c_{5}=1 .
\end{aligned}
$$

The following proposition is proved by a straightforward computation, similar to the proof of Proposition 7.2.

Proposition 7.3. The family of stable curves $\bar{f}: \bar{X} \rightarrow \bar{C}$ defined by (10, 11) is the unique family satisfying the analog of Lemma 7.1 for $D=13$.

\section{The differential equation associated with the Teichmüller curves $W_{17}^{\varepsilon}$} AND $W_{13}$

The goal of this section is to show that the family $\bar{x}^{\varepsilon}(D)$ of curves from $\S \square$ is the universal family corresponding to the Teichmüller curve $\bar{W}_{D}^{\varepsilon}$. The key step is showing the existence of an indigenous bundle on a finite cover of $\bar{W}_{D}^{\varepsilon}$. We only treat the case $D=17$ and $\varepsilon=1$ in detail. The proof for $D=17$ and $\varepsilon=0$ is obtained by replacing $\sqrt{17}$ by $-\sqrt{17}$. The case $D=13$ is similar, and left to the reader.

We let $D=17$ and $\varepsilon=1$, and drop $D$ and $\varepsilon$ from the notation. Let $K=\mathbb{Q}(\sqrt{17})$. Let $\bar{f}: \bar{X} \rightarrow \bar{C}$ be the family of stable curves given by (8, 9) over $K$. The differential forms

$$
\omega_{1}:=\frac{\mathrm{d} x}{y}, \quad \omega_{2}:=\frac{x \mathrm{~d} x}{y}
$$

form a basis of $H^{0}\left(\bar{X}, \Omega \frac{1}{C} / K\right)$. More precisely, $\omega_{i}$ is a section of $\mathcal{L}_{i}=\mathcal{E}_{i}^{(1,0)}$. Recall that $S=\left\{0,1, \infty, \tau, \tau^{-1}\right\}$, where $\tau$ is defined by (17). Write $S^{\prime}=S \backslash\{\infty\}$. Let

$$
\nabla: \mathcal{H}_{\mathrm{dR}}^{1}(\bar{X}) \rightarrow \mathcal{H}_{\mathrm{dR}}^{1}(\bar{X}) \otimes \Omega_{\bar{C} / K}^{1}(\log S)
$$

be the Gauß-Manin connection, and let $t$ be the parameter of $\bar{C}$ chosen in $\S$ 7. We write $\omega^{\prime}=\nabla(\partial / \partial t) \omega_{i}$.

Proposition 8.1. The sections $\omega_{i}$ attached to $W_{17}^{1}$ satisfy the differential equation

$$
\omega_{i}^{\prime \prime}+A_{i}(t) \omega_{i}^{\prime}+B_{i}(t) \omega_{i}=0 \in \mathcal{H}_{\mathrm{dR}}^{1}(\bar{X})
$$


with

$$
A_{1}=\sum_{\tau_{i} \in S^{\prime}} \frac{1}{t-\tau_{i}}, \quad B_{1}=\frac{576 t^{2}+(-4557+891 \sqrt{17}) t+(1296-240 \sqrt{17})}{2^{8} \prod_{\tau_{i} \in S^{\prime}}\left(t-\tau_{i}\right)}
$$

and

$$
A_{2}=\sum_{\tau_{i} \in S^{\prime}} \frac{1}{t-\tau_{i}}-\frac{1}{t-\mu_{1}}-\frac{1}{t-\mu_{2}}, \quad B_{2}=\frac{\mathcal{B}}{2^{14}\left(t-\mu_{1}\right)\left(t-\mu_{2}\right) \prod_{\tau_{i} \in S^{\prime}}\left(t-\tau_{i}\right)},
$$

where

$$
\begin{gathered}
\mathcal{B}=4096 t^{4}+(35616-12768 \sqrt{17}) t^{3}+(-260375+69633 \sqrt{17}) t^{2}+ \\
+(-177536+38528 \sqrt{17}) t+47104-10240 \sqrt{17}
\end{gathered}
$$

and where $\mu_{1}$ and $\mu_{2}$ are the roots of $128 t^{2}+(137-95 \sqrt{17}) t+128=0$.

Proof. A straightforward, but somewhat tedious computation shows that $\omega_{i}^{\prime \prime}+A_{i}(t) \omega_{i}^{\prime}+$ $B_{i}(t) \omega_{i}$ is an exact differential form, for $i=1,2$.

Proposition 8.1 implies that there exists a decomposition $\mathcal{H}_{\mathrm{dR}}^{1}(\bar{X})=\mathcal{E}_{1} \oplus \mathcal{E}_{2}$ of flat vector bundles. We define line bundles $\mathcal{L}_{i}$ on $\bar{C}$ by $\mathcal{L}_{i}=\mathcal{E}_{i}^{(1,0)}$. By definition, $\omega_{i}$ is a section of $\mathcal{L}_{i}$.

Lemma 8.2. The Kodaira-Spencer map $\Theta: \mathcal{L}_{1} \rightarrow\left(\mathcal{E}_{1} / \mathcal{L}_{1}\right) \otimes \Omega \frac{1}{C}(\log S)$ is an isomorphism, i.e. $\mathcal{E}_{1}$ is an indigenous bundle.

Proof. At points of $C$, i.e. where the fiber of $f$ is smooth, the differential equation (12) has no singularities. Consequently, the Kodaira-Spencer map does not vanish at those points (9] Proposition 2.2(b)). The nonvanishing of the Kodaira-Spencer map at points in $S$ is shown in $([9]$, Proposition 2.2(d)).

Proposition 8.3. The family $\bar{x}$ of stable curves is the universal family corresponding to the Teichmüller curve $W_{17}^{1}$.

Proof. Since $\mathcal{E}_{1}$ is an indigenous bundle on $\bar{C}$, it follows from 24, Proposition 5.5, that $\bar{C}$ is the cover of a Teichmüller curve. Since the moduli map $\bar{C} \rightarrow \overline{\mathcal{M}}_{2}$ factors through $\bar{W}_{17}^{1}$, the proposition follows.

Recall that $\bar{C} \rightarrow \bar{W}_{17}^{\varepsilon}$ is a degree-2 cover given by $s=\left(t^{2}+1\right) / 2 t$. We denote by $\Sigma=\left\{1,-1, \infty,\left(\tau^{2}+1\right) / 2 \tau\right\}$ the union of the set of cusps with the elliptic point on $\bar{W}_{17}^{\varepsilon}$.

Corollary 8.4. The pointed curve $\left(\bar{W}_{17}^{\varepsilon}, \Sigma\right)$ may not be defined over $\mathbb{Q}$.

Proof. It suffices to check that the $j$-invariant of $\Sigma$ is not in $\mathbb{Q}$.

We finish this section by giving a formula for the differential equations corresponding to $W_{13}$. Recall that the set of cusps is in this case $S=\left\{0,1, \infty, \rho, \rho^{-1}\right\}$, where $\rho$ is given by (18). Let $S^{\prime}=S \backslash\{\infty\}$. Let $\mu_{1}, \mu_{2}$ be the roots of $t^{2}+(5+28 \sqrt{13}) t / 48+1=0$.

Proposition 8.5. The sections $\omega_{i}$ attached to $W_{13}$ satisfy the differential equation

$$
\omega_{i}^{\prime \prime}+A_{i}(t) \omega_{i}^{\prime}+B_{i}(t) \omega_{i}=0 \in \mathcal{H}_{\mathrm{dR}}^{1}(\bar{X})
$$

with

$$
A_{1}=\sum_{\tau_{i} \in S^{\prime}} \frac{1}{t-\tau_{i}}, \quad B_{1}=\frac{1152 t^{2}+(333 \sqrt{13}-576) t-120 \sqrt{13}+192}{2^{9} \prod_{\tau_{i} \in S^{\prime}}\left(t-\tau_{i}\right)}
$$


and

$$
\begin{aligned}
& A_{2}=\sum_{\tau_{i} \in S^{\prime}} \frac{1}{t-\tau_{i}}-\frac{1}{t-\mu_{1}}-\frac{1}{t-\mu_{2}}, \\
& B_{2}=\frac{98304 t^{4}+(135936 \sqrt{13}+67584) t^{3}+(698944+5744 \sqrt{13}) t^{2}-80181+21234 \sqrt{13}}{2^{17} 3\left(t-\mu_{1}\right)\left(t-\mu_{2}\right) \prod_{t \in S^{\prime}}\left(t-\tau_{i}\right)} .
\end{aligned}
$$

The proofs of Lemma 8.2 and Proposition 8.3 immediately carry over to this situation, and we conclude that (10, 11) define the universal family of stable curves of genus 2 corresponding to the Teichmüller curve $W_{13}$.

Remark 8.6. Let $\Sigma=\left\{ \pm 1, \infty,\left(\rho^{2}+1\right) / 2 \rho\right\} \subset \bar{W}_{13}$. Then the $j$-invariant of $\Sigma$ is indeed in $\mathbb{Q}$, as also follows from Theorem 3.3 .

\section{INTEGRAL SOLUTIONS OF DIFFERENTIAL EQUATIONS}

The expansions of the solution $u_{i}$ of the differential operators $L_{i}$ from (12) at $t=0$ are given by

$$
\begin{aligned}
& u_{1}=1+\frac{81-15 \sqrt{17}}{2^{4}} t+\frac{4845-1155 \sqrt{17}}{2^{6}} t^{2}+\frac{3200225-775495 \sqrt{17}}{2^{11}} t^{3}+\cdots, \\
& u_{2}=1+\frac{23-5 \sqrt{17}}{2^{3}} t+\frac{5561-1343 \sqrt{17}}{2^{7}} t^{2}+\frac{452759-109793 \sqrt{17}}{2^{9}} t^{3}+\cdots
\end{aligned}
$$

On the other hand, the coefficients of $u_{i}$ are determined by the recursion formula (15) below which causes a division by $(j+1)^{2}$ in the $j$-th step. The purpose of this section is to explain why nevertheless almost no denominators occur. This rare phenomenon also occurs in Apéry's differential equation ([5]). In Apéry's case the integrality of the coefficients is shown by guessing a closed formula. This seems much harder in our case. Instead we show that $L_{i}$ admits an integral solution $\left(\bmod \wp^{n}\right)$ for all $n$. This relies on a result of Katz ([16]) which states that the expansion coefficients of $\omega_{i}$ are solutions of $L_{i}$ modulo $p^{n}$.

Our strategy works more generally. We fix $D$ and $\varepsilon \in\{0,1\}$, and let $\bar{W}_{D}^{\varepsilon}$ be the corresponding Teichmüller curve. We exclude the Teichmüller curve corresponding to $(X, \omega) \in \Omega \mathcal{M}_{2}(1,1)$. This is no restriction, as the affine group in this case is a triangle group, and the results of this section are well-understood in that case.

Let $\bar{C} \rightarrow \bar{W}_{D}^{\varepsilon}$ be a cover which is only branched at the cusps and elliptic points of $\bar{W}_{D}^{\varepsilon}$, such that $\bar{C}$ does not have any elliptic points. Such a cover always exists. In the case that $D=13,17$, the cover $\bar{C} \rightarrow \bar{W}_{D}^{\varepsilon}$ is the degree-2 cover of $\oint 7$. In this section, we consider the case that $g(\bar{C})=0$.

The assumption that $g(\bar{C})=0$ implies that there exist differential operators $L_{i}$ on $\bar{C}$ corresponding to the flat vector bundles $\mathcal{E}_{i}$ on $\bar{C}$ (compare to the discussion in $\S(5)$ ). In the case that $D=13,17$ these are the differential operators determined in $\S 8$. The singularities of $L_{1}$ are exactly the cusps of $\bar{C}$. Moreover, since the genus of $\bar{C}$ is zero, we may choose a coordinate $t$ on $\bar{C}$ such that $t=0, \infty$ are cusps. The singularities of $L_{2}$ are the cusps together with the zeros of the Kodaira-Spencer morphism of $\mathcal{E}_{2}$.

We assume, moreover, that the local exponents of $L_{i}$ at all singularities, except possibly $t=\infty$, are integers. After replacing $L_{i}$ by an equivalent differential operator, we may therefore assume that the local exponents of $L_{i}$ at the cusps $t \neq \infty$ are $(0,0)$, and that the local exponents of $L_{2}$ at the zeros of the Kodaira-Spencer map are $\left(0, \delta_{j}\right)$, with $\delta_{j} \geq 0$ (in Lemma 9.2 , we give a more precise statement). In the case that $D=13,17$ the differential 
operators $L_{i}$ computed in $\S 8$ satisfy these conditions. Moreover, one checks using [21, Theorem 9.8] that the conditions are also satisfied for $D=21,29,33$. Before stating the main result of this section, we need to introduce some notation. As usual, we drop $D$ and $\varepsilon$ from the notation.

Let $R$ be a finite extension of $\mathcal{O}_{D}$ over which the family $f: X \rightarrow C$ can be defined. For a set $\mathcal{S}$ of primes, we denote by $R_{\mathcal{S}}$ the set of $\mathcal{S}$-integers in $R$. There exists a finite set $\mathcal{S}$ of primes of $R$ and a model $\bar{f}_{\mathcal{S}}: \bar{X}_{R_{\mathcal{S}}} \rightarrow \overline{\mathcal{C}}_{R_{\mathcal{S}}}$ of $f$ such that for every prime $\wp$ which is invertible in $R_{\mathcal{S}}$ the reduction $\bar{f}_{\mathcal{S}} \otimes_{R_{\mathcal{S}}} R / \wp$ of $\overline{f_{\mathcal{S}}}$ modulo $\wp$ is a family of stable curves of genus 2 with the same number of degenerate fibers as $\bar{f}$. More precisely, we require that the set, $S_{i}$, of singularities of $L_{i}$ extends to an étale divisor over $\operatorname{Spec}\left(R_{\mathcal{S}}\right)$. (This may be accomplished by extending the set $\mathcal{S}$, if necessary.)

In $\S$ 10, we explicitly determine such a set $\mathcal{S}$ in the case that $D=13$ or $D=17$. We remark that the set $\mathcal{S}$ depends on the choice of a model for $\bar{X}$.

It follows from Lemma 5.3 that $L_{i}$ has a unique holomorphic solution $u_{i} \in K[[t]]$ with $u_{i}(t=0)=1$. Here one uses that $t=0$ is a cusp of $L_{i}$, and that the local exponents of $L_{i}$ at $t=0$ are $(0,0)$. The goal of this section is to prove the following theorem.

Theorem 9.1. The unique holomorphic solution $u_{i}$ of $L_{i}$ with $u_{i}(t=0)=1$ has coefficients in $R_{\mathcal{S}}[[t]]$.

As a first step in the proof we determine the recursion relation satisfied by the coefficients of $u_{i}$.

Write $u_{i}=\sum_{j \geq 0} u_{j}^{(i)} t^{j}$. We let $S_{i}$ be the set of singularities of $L_{i}$, and put $r_{i}=\left|S_{i}\right|$. We write $\left(\gamma_{i}, \gamma_{i}\right)$ for the local exponents of $L_{i}$ at $t=\infty$. Let $M_{i}=\prod_{\tau \neq 0, \infty} \tau$ be the product of the singularities of $L_{i}$ different from $0, \infty$. In the case that the differential operator $L_{i}$ is given explicitly, these invariants may be read off from the explicit expression for $L_{i}$.

Recall that $r_{1}$ is the number of cusps of $\bar{C}$, and $r_{2}=r_{1}+\mu$, where $\mu$ is the number of zeros of the Kodaira-Spencer morphism of $\mathcal{E}_{2}$. In the case that $D=13,17$, we have that $r_{1}=5$ and $r_{2}=7$. The following lemma expresses the local exponent $\gamma_{i}$ at $\infty$ in terms of known invariants. Recall that $\lambda_{2}$ is the Lyapunov exponent which was introduced in $\S 2$,

Lemma 9.2. (a) We have $\gamma_{1}=\left(r_{1}-2\right) / 2$.

(b) Let $\mu_{j}$ be a zero of the Kodaira-Spencer map $\Theta$ of $L_{2}$ and let $\delta_{j}$ the order of vanishing of $\Theta$ at $\mu_{j}$. Then the local exponents of $L_{2}$ at $t=\mu$ are $\left(0, \delta_{j}+1\right)$.

(c) The local exponent of $L_{2}$ at $t=\infty$ satisfies

$$
\gamma_{2}=\lambda_{2}\left(r_{1}-2\right) / 2 \text {. }
$$

Proof. Part (a) follows from the Riemann relation together with the assumption that the local exponents of $L_{1}$ at the cusps different from $\infty$ are $(0,0)$. Part (b) is proved in [9], Proposition 2.2.(b).

Let $\left\{\mu_{j}\right\}$ be the set of zeros of $\Theta$, and let $\delta_{j}$ be the order of vanishing of $\Theta$ at $\mu_{j}$. Then

$$
\sum_{j} \delta_{j}=2 g(\bar{C})-2+\left|S_{1}\right|-\lambda_{2}\left(r_{1}-2\right)=\left(r_{1}-2\right)\left(1-\lambda_{2}\right)
$$

Therefore the Riemann relation, together with (b) and our assumption on the local exponents, implies that

$$
2 \gamma_{2}=-\sum_{j}\left(\delta_{j}+1\right)+\left|S_{2}\right|-2=r_{1}+\mu-2 \sum_{j} \delta_{j}-\mu=\lambda_{2}\left(r_{1}-2\right) .
$$

This proves the lemma. 
In the case that $D=13,17$, the Kodaira-Spencer morphism of $L_{2}$ has two simple zeros. Lemma 9.2 implies in this case that $\gamma_{1}=3 / 2$ and $\gamma_{2}=1 / 2$. Moreover, $M_{1}=1 \cdot \tau \cdot \tau^{-1}=1$ and $M_{2}=M_{1} \cdot \mu_{1} \cdot \mu_{2}=1$, as follows from the formulas in $\S 7$ and $\S 8$, It might be possible to show, using Theorem 3.3 , that one may choose a parameter $t$ on $\bar{C}$ such that this holds in general.

Lemma 9.3. The coefficients $u_{j}^{(i)}$ of $u_{i}$ satisfy a recursion

$$
D_{j,-1} u_{j+1}^{(i)}+D_{j, 0} u_{j}^{(i)}+\cdots+D_{j, r_{i}-3} u_{j-r_{i}+3}^{(i)}=0,
$$

where $D_{j,-1}= \pm(j+1)^{2} M_{i}$ and $D_{j, r_{i}-3}=\left(j+\gamma_{i}-r_{i}+3\right)^{2}$.

Proof. It is easy to see that the coefficients of a solution of a Fuchsian differential equation satisfy a recursion as in (15). The order of the recursion depends on our normalization of the local exponents which assures that the numerator of $A_{i}$ (resp. $B_{i}$ ) has degree $r_{i}-1$ (resp. $r_{i}-2$ ) in $t$. The formula for $D_{j,-1}$ and $D_{j, r_{i}-2}$ follows from an easy calculation, using the assumption that the local exponents at $t=0$ are $(0,0)$.

In the rest of this section, we fix a prime $p \notin \mathcal{S}$ and let $\wp \mid p$ be a prime of $R_{\mathcal{S}}$. We let $k=1$ if $D$ is a quadratic residue $(\bmod p)$, and $k=2$ otherwise, i.e. $R_{\mathcal{S}} / \wp=\mathbb{F}_{p^{k}}$. We write $\mathbb{Z}_{p^{k}}=W\left(\mathbb{F}_{p^{k}}\right)$ for the $\wp$-adic completion of $R_{\mathcal{S}}$. Let $\mathcal{R}$ be the $\wp$-adic completion of

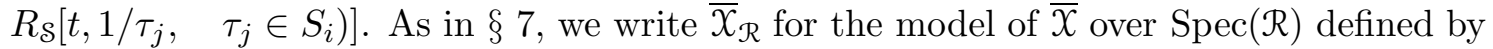
(8). We write $\bar{x}_{\wp}:=\bar{x}_{\mathcal{R}} \otimes_{\mathcal{R}}(\mathcal{R} / \wp)$.

As in $\S$ [, we let

$$
y^{2}=g_{t}(x)=\sum_{k=0}^{5} c_{k} x^{k}
$$

be an equation for $\bar{x}$. The results of $\S 2$ imply that $\operatorname{deg}_{t}\left(c_{k}\right)=\left(r_{1}-2\right)(5-k) / 3$. The definition of $\bar{C}$ implies that these are integers.

Definition 9.4. Let $n$ be a natural number. We define polynomials $B_{n, 1} \in R_{\mathcal{S}}[t]$ (resp. $\left.B_{n, 2} \in R_{\mathcal{S}}[t]\right)$ as the coefficient of $x^{p^{n}-1}$ (resp. $\left.x^{2 p^{n}-1}\right)$ in $g^{\left(p^{n}-1\right) / 2}$. Similarly, we define $C_{n, 1} \in R_{\mathcal{S}}[t]$ (resp. $\left.C_{n, 2} \in R_{\mathcal{S}}[t]\right)$ as the coefficient of $x^{p^{n}-2}\left(\right.$ resp. $x^{2 p^{n}-2}$ ) in $g^{\left(p^{n}-1\right) / 2}$.

Lemma 9.5. (a) The polynomials $B_{n, 1}$ and $B_{n, 2}$ are solutions $\left(\bmod p^{n}\right)$ of $L_{1}$.

(b) The polynomials $C_{n, 1}$ and $C_{n, 2}$ are solutions $\left(\bmod p^{n}\right)$ of $L_{2}$.

Proof. We note that $x$ is a local parameter of $\bar{x}_{\wp}$ at $x=0$, except at the 5 zeros of $c_{0}$. We write

$$
\omega_{1}=\frac{\mathrm{d} x}{y}=g^{\left(p^{n}-1\right) / 2} \frac{\mathrm{d} x}{y^{p^{n}}}=\sum_{m \geq 0} P_{m} x^{m} \frac{\mathrm{d} x}{y^{p^{n}} x},
$$

and

$$
\omega_{2}=\frac{x \mathrm{~d} x}{y}=g^{\left(p^{n}-1\right) / 2} \frac{x \mathrm{~d} x}{y^{p^{n}}}=\sum_{m \geq 0} Q_{m} x^{m} \frac{\mathrm{d} x}{y^{p^{n}} x},
$$

where $P_{m}$ (resp. $Q_{m}$ ) is the coefficient of $x^{m-1}$ (resp. $\left.x^{m-2}\right)$ in $g^{\left(p^{n}-1\right) / 2}$. In particular $P_{m}, Q_{m} \in \mathcal{R}$.

The coefficients $P_{m}$ and $Q_{m}$ are called the expansion coefficients of $\omega_{i}$. Katz ([16]) shows that $P_{m}$ (resp. $Q_{m}$ ) is a solution of $L_{1}$ (resp. $L_{2}$ ) modulo $m$, i.e. of the same differential equation which is satisfied by $\omega_{1}$ (resp. $\omega_{2}$ ). One may check this also directly, by noting that $(\partial / \partial t) y^{p^{n}} \equiv 0\left(\bmod p^{n}\right)$. Since $B_{n, k}=P_{k p^{n}}$ and $C_{n, k}=Q_{k p^{n}}$, it follows that these polynomials are solutions of $L_{1}$ and $L_{2}$ (modulo $p^{n}$ ), as well. 
Remark 9.6. By using the formula for $\operatorname{deg}_{t}\left(c_{k}\right)$, one shows that the degree of $B_{n, 1}$ and $B_{n, 2}$ is less than or equal to $d_{n, 1}:=\left(r_{1}-2\right)\left(p^{n}-1\right) / 2$ and $d_{n, 2}:=\left(r_{1}-2\right)\left(p^{n}-3\right) / 6$, respectively. Similarly, the degree of $C_{n, 1}$ and $C_{n, 2}$ is less than or equal to $e_{n, 1}:=\left(r_{1}-2\right)\left(3 p^{n}-1\right) / 6$ and $e_{n, 2}:=\left(r_{1}-2\right)\left(p^{n}-1\right) / 6$, respectively. In Remark 9.10 we show that equality holds.

Lemma 9.7. $\quad$ (a) Suppose that $D$ is a quadratic residue $(\bmod p)$. Then

$$
B_{1,2} \equiv C_{1,1} \equiv 0 \quad(\bmod p) .
$$

(b) Suppose that $D$ is a quadratic nonresidue $(\bmod p)$. Then

$$
B_{1,1} \equiv C_{1,2} \equiv 0 \quad(\bmod p) .
$$

Proof. We consider $\omega_{j}$ as element of $\mathcal{E}_{j} \otimes_{\mathcal{R}}(\mathcal{R} / \wp) \subset \mathcal{H}_{\mathrm{dR}}\left(\bar{X}_{\mathcal{R}}\right) \otimes_{\mathcal{R}}(\mathcal{R} / \wp)$. We denote by $\mathcal{C}$ the Cartier operator, and compute that

$$
\mathcal{C} \omega_{1}=\mathcal{C} \frac{x g^{(p-1) / 2}}{y^{p}} \frac{\mathrm{d} x}{x}=\left[B_{1,1}^{1 / p}+B_{1,2}^{1 / p} x\right] \frac{\mathrm{d} x}{y}, \quad \mathcal{C} \omega_{2}=\mathcal{C} \frac{x^{2} g^{(p-1) / 2}}{y^{p}} \frac{\mathrm{d} x}{x}=\left[C_{1,1}^{1 / p}+C_{1,2}^{1 / p} x\right] \frac{\mathrm{d} x}{y} .
$$

For the definition and properties of the Cartier operator, we refer to the article of Illusie in [4].

Suppose that $D$ is a quadratic residue $(\bmod p)$. Then $\mathcal{C}$ stabilizes $\mathcal{L}_{j} \subset \mathcal{E}_{j}$. Therefore $B_{1,2} \equiv C_{1,1} \equiv 0(\bmod p)$.

If $D$ is a quadratic nonresidue $(\bmod p)$, the Cartier operator sends $\mathcal{L}_{1} \subset \mathcal{E}_{1}$ to $\mathcal{L}_{2} \subset \mathcal{E}_{2}$, and conversely. This implies that $B_{1,1} \equiv C_{1,2} \equiv 0(\bmod p)$.

Lemma 9.8. (a) Suppose that $(D / p)=1$. Then

$$
B_{n+1,1} \equiv B_{n, 1}^{p} B_{1,1} \quad(\bmod p), \quad C_{n+1,2} \equiv C_{n, 2}^{p} C_{1,2} \quad(\bmod p) .
$$

In particular, $B_{n, 1} \equiv B_{1,1}^{p^{n-1}+\cdots+p+1}(\bmod p)$ and $C_{n, 2} \equiv C_{1,2}^{p^{n-1}+\cdots+p+1}(\bmod p)$.

(b) Suppose that $(D / p)=-1$. Then

$$
B_{n+1,2} \equiv C_{n, 1}^{p} B_{1,2} \quad(\bmod p), \quad C_{n+1,1} \equiv B_{n, 2}^{p} C_{1,1} \quad(\bmod p) .
$$

In particular, $B_{n, 2} \equiv C_{1,1}^{\cdots+p^{3}+p} \cdot B_{1,2}^{\cdots+p^{2}+1}(\bmod p)$ and $C_{n, 1} \equiv C_{1,1}^{\cdots+p^{2}+1} \cdot B_{1,2}^{\cdots+p^{3}+p}$ $(\bmod p)$.

Proof. We note that

$$
g^{\left(p^{n+1}-1\right) / 2}=\left(g^{p}\right)^{\left(p^{n}-1\right) / 2} \cdot g^{(p-1) / 2} .
$$

The definition of the $B_{n, i}$ implies therefore that

$$
B_{n+1,1} \equiv B_{n, 1}^{p} \cdot B_{1,1}+B_{n, 2}^{p} \cdot B_{1,2} \quad(\bmod p) .
$$

We used here that $\operatorname{deg}\left(g^{(p-1) / 2}\right)=5(p-1) / 2$. Assume now that $(D / p)=1$. Lemma 9.7 implies that $B_{1,2} \equiv 0(\bmod p)$. Part (a) of the lemma follows immediately. The other statements follow similarly.

Lemma 9.9. Let $k=1$ if $(D / p)=1$ and $k=2$ if $(D / p)=-1$. The polynomials $B_{n, k}$ and $C_{n, 2-k}$ are not identically zero (modulo $p^{n}$ ).

Proof. Lemma 9.8 implies that it suffices to prove the lemma for $n=1$. Let $t=t_{i} \neq \infty$ be a cusp. Denote by $\bar{x}_{i, \wp}$ the reduction of the degenerate fiber at $t=t_{i}$. Lemma 2.1 implies that every irreducible component of $\bar{X}_{i, \wp}$ has geometric genus 0 . It is well known that this implies that $\bar{X}_{i, \wp}$ is ordinary. (See for example [8], Lemma 1.3.) Therefore the matrix of the Frobenius morphism $F: H^{1}\left(\bar{X}_{\wp}, \mathcal{O}\right) \rightarrow H^{1}\left(\bar{X}_{\wp}, \mathcal{O}\right)$ is invertible at $t=t_{i}$. Since the 
Cartier operator is the transpose of the Frobenius under Serre duality, we conclude that for $t_{i} \neq \infty$ we have that $B_{1,1}\left(t_{i}\right) \not \equiv 0(\bmod p)$.

Remark 9.10. The proof of Lemma 9.9 implies that the polynomials $B_{n, k}$ and $C_{n, 2-k}$ do not vanish at $t=t_{i}\left(\bmod p^{n}\right)$ for all $i$ such that $t_{i} \neq \infty$. An analogous argument, after replacing $t$ by a local parameter $1 / t$ at $t=\infty$, implies that the polynomial $B_{n, k}$ (resp. $\left.C_{n, 2-k}\right)$ has degree $d_{n, k}\left(\right.$ resp. $\left.e_{n, 2-k}\right) \quad\left(\bmod p^{n}\right)$. Here $d_{n, k}$ and $e_{2-k}$ are defined in Remark 9.6 .

We now apply Lemma 9.3 to the operator $L_{1}$. We write

$$
B_{n, k}=\sum_{i \geq 0} v_{i}^{(n)} t^{i}
$$

Since $B_{n, k}$ is a solution of $L_{1}\left(\bmod p^{n}\right)$, the coefficients $v_{i}^{(n)}$ satisfy the recursion (15) $\left(\bmod p^{n}\right)$.

Lemma 9.11. Let $N=\lceil n / 2\rceil$. Let $\beta_{n}$ be the smallest integer such that $v_{j}^{(n)} \equiv 0\left(\bmod p^{n}\right)$ for $j=\beta_{n}+1, \beta_{n}+2, \ldots, \beta_{n}+r_{1}-2$. Then $\beta_{n} \equiv-\gamma_{1} \equiv-\left(r_{1}-2\right) / 2\left(\bmod p^{N}\right)$. In particular,

$$
\beta_{n} \geq \begin{cases}\frac{p^{N}-r_{1}+2}{2} & \text { if } r_{1} \text { is odd } \\ p^{N}-\frac{r_{1}-2}{2} & \text { if } r_{1} \text { is even. }\end{cases}
$$

Proof. The definition of $\beta_{n}$ implies that $v_{\beta_{n}}^{(n)} \not \equiv 0\left(\bmod p^{n}\right)$. Therefore the recursion (15) implies that $D_{\beta_{n}+r_{1}-3, r_{1}-3} \equiv 0\left(\bmod p^{n}\right)$. The statement of the lemma follows now immediately from the formula for $D_{\beta_{n}+r_{1}-3, r_{1}-3}$ and the fact that $\beta_{n} \geq 0$.

Remark 9.12. The proof of Lemma 9.11 implies that $\sum_{i=0}^{\beta_{n}} v_{j}^{(n)} t^{i}$ is also a solution $\left(\bmod p^{n}\right)$, since its coefficients satisfy the recursion (15) $\left(\bmod p^{n}\right)$. Since $v_{\beta_{n}}^{(n)} \not \equiv 0$ $\left(\bmod p^{n}\right)$, it follows that $L_{1}$ has a solution $\left(\bmod p^{n}\right)$ of degree $\beta_{n}$. The inequality for $\beta_{n}$ in Lemma 9.11 gives therefore a lower bound on the degree of a polynomial solution $\left(\bmod p^{n}\right)$ of $L_{1}$.

For the proof of Theorem 9.1, we do not need to know this. We only need to know that there exists a polynomial solution (modulo $p^{n}$ ) of degree $\beta_{n}$, and that $\lim _{n \rightarrow \infty} \beta_{n}=\infty$.

Proposition 9.13. For every $j \geq 0$, we have that $u_{j}^{(1)} \in \mathbb{Z}_{p^{k}}$.

Proof. We first fix an integer $n$. Since $B_{n, k}(0) \not \equiv 0\left(\bmod p^{n}\right)($ Lemma 9.9) , the polynomial $B_{n, k}^{\prime}:=B_{n, k} / B_{n, k}(0)$ also has coefficients in $\mathbb{Z}_{p^{k}}$. The coefficients of $B_{n, k}^{\prime}$ still satisfy (15). We also denote these coefficients by $v_{j}^{(n)}$. The definition of $\beta_{n}$ implies that for $0 \leq j \leq \beta_{n}$ the coefficients $v_{j}^{(n)} \in \mathbb{Z}_{p^{k}}$ are uniquely determined $\left(\bmod p^{n}\right)$ by $v_{0}^{(n)}=1$ and (15)). Since the $u_{j}^{(1)}$ satisfy the same recursion, we conclude that

$$
u_{j}^{(1)} \equiv v_{j}^{(n)} \quad\left(\bmod p^{n}\right) .
$$

In particular, we conclude that $u_{j}^{(1)} \in \mathbb{Z}_{p^{k}}$ for all $j \leq \beta_{n}$.

For every $j \geq 0$, there exists an $n$ such that $j \leq \beta_{n}$ (Lemma 9.11). Since $u_{j}^{(1)} \in K$, the proposition follows. 
Now we apply Lemma 9.3 to the solution $u_{2}$ of $L_{2}$ which has $r_{2}=r_{1}+\mu$ singularities. Recall from $\$ 9$ that $C_{n, 2-k} \in R_{\mathcal{S}}[t]$ is a solution $\left(\bmod p^{n}\right)$ of $L_{2}$ of degree $e_{n, 2-k}$. We write

$$
C_{n, k}=\sum_{j=0}^{e_{n, k}} w_{j}^{(n)} t^{j} .
$$

Note that the $w_{j}^{(n)}$ satisfy the recursion (15) $\left(\bmod p^{n}\right)$.

Let $\gamma_{n}$ be the smallest integer such that $w_{j}^{(n)} \equiv 0\left(\bmod p^{n}\right)$ for $j=\gamma_{n}+1, \ldots \gamma_{n}+r_{2}-2$. The recursion (15) implies that $D_{\gamma_{n}+r_{2}+3, r_{2}-3} \equiv 0\left(\bmod p^{n}\right)$. This proves the following lemma which is an analog of Lemma 9.11, by using the expression for the local exponent of $L_{2}$ at $\infty$ we gave in Lemma 9.2.

Lemma 9.14. Let $N=\lceil n / 2\rceil$. Then $\gamma_{n} \equiv-\gamma_{2}\left(\bmod p^{N}\right)$. In particular,

$$
\gamma_{n} \geq \frac{p^{N}-\left(r_{1}-2\right) \lambda_{2}}{2}
$$

Note that the estimate for $\gamma_{n}$ need not be an integer, so one may improve it a bit, depending on the values of $r_{1}$ and $\lambda_{2}$. However, we do not need this. The proof of Proposition 9.13 caries now over to $L_{2}$.

Proposition 9.15. For every $j \geq 0$, we have that $u_{j}^{(2)} \in \mathbb{Z}_{p^{k}}$.

Proof of Theorem 9.1. The theorem follows immediately from Propositions 9.13 and 9.15 ,

\section{Reduction of the families $X^{\varepsilon}(D)$ to Characteristic $p>0$}

Let $\mathcal{O}_{D} \subset \mathbb{Q}(\sqrt{D})$ be an order of discriminant $D$ and $R=\mathcal{O}_{D}[1 / 2]$. Let $p \neq 2$ be a prime number, and let $\wp$ be a prime of $R$ above $p$. We denote by $R_{\wp}$ the completion of $R$ at $\wp$. We say that $\bar{f}: \bar{x} \rightarrow \bar{C}$ has good reduction at $\wp$ if there exists a model $\bar{f}_{R}: \bar{y}_{R} \rightarrow \overline{\mathrm{e}}$ of $\bar{f}: \bar{X} \rightarrow \bar{C}$ over $\operatorname{Spec}\left(R_{\wp}\right)$ such that $\bar{f}_{R} \otimes_{R}(R / \wp)$ is a family of stable curves of genus 2 with $|S|$ singular fibers. We say that $\bar{f}$ has potentially good reduction if such a model exists after replacing $R$ by the completion of a finite extension.

Let $D \in\{13,17\}$ and $\varepsilon \in\{0,1\}$. In this section we consider the reduction to characteristic $p>0$ of the stable families of curves $\bar{X}^{\varepsilon}(D)$ defined by (8, 9) and (10, 11), respectively. This section should be considered as a complement to the results in the previous sections, though it is logically independent of it.

Recall that it follows from the general theory that the family $\bar{X}^{\varepsilon}(D)$ has good reduction at all but a finite set of primes. In this section, we determine this set for $D=13,17$. This proof relies on the explicit equation for the family of curves $\bar{X}^{\varepsilon}(D)$. One expects a similar result to hold much more generally. It would be interesting to give a geometric proof of the results of this section, relying on the properties of Teichmüller curves. This would give a much deeper insight into the arithmetic properties of Teichmüller curves. If $D$ and $\varepsilon$ are understood, we drop them from the notation.

Since the coefficients of $f_{D}$ are in $R$, the formulas (8) and (10) define a model $\bar{X}_{R}$ of $\bar{X}$ over $\operatorname{Spec}(R)$. One may ask whether this model already reduces to a family of stable curves of genus 2 with $|S|$ singular fibers. In this section, we consider this question in the case that $D \in\{13,17\}$. 
Proposition 10.1. (a) Let $D=17$. Let $p \neq 2,17$ be a prime number, and let $\wp \mid p$ be a prime of $R$ above $p$. Then $\bar{f}$ has good reduction at $\wp$.

(b) Let $D=13$. Let $p \neq 2,3,13$ be a prime number, and let $\wp \mid p$ be a prime of $R$ above $p$. Then $\bar{f}$ has good reduction at $\wp$.

Proof. We first consider the case $D=17$. Let $\wp$ be as in the statement of the lemma. One computes that the discriminant of $g_{17}(x)=\sum_{k} c_{k} x^{k}$ is equal to

$$
-\frac{17^{10}}{2^{12}}(4+\sqrt{17})^{19}\left(\frac{5}{2}+\frac{1}{2} \sqrt{17}\right)\left(\frac{5}{2}-\frac{1}{2} \sqrt{17}\right)^{18}(2 t-31+7 \sqrt{17})^{3}(64 t-31-7 \sqrt{17})^{3}(t-1)^{4} t^{5} .
$$

Since $N(4+\sqrt{17})=1$ and $N((5+\sqrt{17}) / 2)=N((5-\sqrt{17}) / 2)=2$, it follows that $\bar{X} \otimes_{R}(R / \wp)$ is a curve of genus 2 for generic $t$.

Choose

$$
\tau=\frac{31-7 \sqrt{17}}{2}, \quad \tau^{-1}=\frac{31+7 \sqrt{17}}{64} .
$$

Then $S=\left\{0,1, \infty, \tau, \tau^{-1}\right\}$ is the set of cusps of $C$. We claim that the points $0,1, \infty, \tau, \tau^{-1}$ are pairwise noncongruent $(\bmod \wp)$. Namely, one computes that for every pair $P_{1}, P_{2}$ of points we have that $N\left(P_{1}-P_{2}\right)$ is a power of 2 . This proves the lemma for $D=17$.

Now let $D=13$ and let $\wp$ be a prime of $R=R(13)$ above $p \neq 2,3,13$. The discriminant of $g_{13}=\sum_{k} d_{k} x^{k}$ is equal to

$$
-\frac{3^{12} 13^{10}}{2^{60}}\left(-\frac{3}{2}+\frac{1}{2} \sqrt{13}\right)^{30}\left(\frac{1}{2}+\frac{1}{2} \sqrt{13}\right)^{6} t^{4}\left(128 t^{2}+71 \sqrt{13} t+128\right)^{4}(t-1)^{4} .
$$

Since $N((-3+\sqrt{13}) / 2)=-1$ and $N((1+\sqrt{13}) / 2)=-3$, it follows that $\bar{X} \otimes_{R}(R / \wp)$ is a curve of genus 2 for generic $t$.

The set of cusps is $\left\{0,1, \infty, \rho, \rho^{-1}\right\}$ with

$$
\rho=-\frac{71}{256} \sqrt{13}+\frac{1}{256} \sqrt{-3}, \quad \rho^{-1}=-\frac{71}{256} \sqrt{13}-\frac{1}{256} \sqrt{-3} .
$$

The proposition for $D=13$ now follows as for $D=17$.

For $D=13,17$, we let $\mathcal{S}_{D} \subset \mathbb{Z}$ be the set of primes such that the the model defined by (8, 9) and (10, 11) defines a family of stable curves with $|S|=5$ singular fibers. Proposition 10.1 implies that $\mathcal{S}_{D}=\{2,3,13\}$ if $D=13$ and $\mathcal{S}_{D}=\{2,17\}$ if $D=17$. We call $\mathcal{S}_{D}$ the set of exceptional primes of the model $\bar{X}_{R}$. Note that $\mathcal{S}_{D}$ depends on the choice of the model.

In the rest of this section, we discuss some partial results on the reduction of $\bar{f}: \bar{X} \rightarrow \bar{C}$ at the exceptional primes $p \neq 2$. A more detailed description might allow one to extend the proof of the integrality to the exceptional primes $p \neq 2$.

Proposition 10.2. Let $D \in\{13,17\}$. The family of curves $\bar{f}^{\varepsilon}(D): \bar{x}^{\varepsilon}(D) \rightarrow \bar{C}(D)$ has potentially good reduction at $p=D$.

Proof. We only discuss the case $D=p=17$ and $\varepsilon=1$. The argument in the other cases is the same. Let $\wp=(\sqrt{p})$ be the unique prime of $R$ above $p$. We remark that $g_{D}^{\varepsilon}(x) \equiv(x+4 t+4)^{5}(\bmod \wp)$. Substituting $x=z-4(t+1)$ yields $g_{D}^{\varepsilon}(z) \equiv z^{5}(\bmod \wp)$. By considering the Newton polygon of $g_{D}^{\varepsilon}(z)$, we find that $g_{D}^{\varepsilon}(z)$ has one root of valuation $v(p)$, and 4 roots of valuation $v(\sqrt{p})$. Therefore we substitute $z=\sqrt{p} w$ and compute

$$
\tilde{g}_{D}^{\varepsilon}(w):=g_{D}^{v} e(w) / p^{3 / 2} \equiv 3\left(t^{2}+3 t+1\right)\left(t^{2}+7 t+1\right) w+5\left(t^{2}+3 t+1\right) w^{3}+w^{5} .
$$


Performing the corresponding coordinate substitution for $y$ as well, one obtains the equation

$$
v^{2}=\tilde{g}_{D}^{\varepsilon}(w)=3\left(t^{2}+3 t+1\right)\left(t^{2}+7 t+1\right) w+5\left(t^{2}+3 t+1\right) w^{3}+w^{5}
$$

which defines a model of $\bar{X}^{\varepsilon}(D)$ over $R_{p}\left[p^{1 / 4}\right]$ whose fiber at $\wp$ is smooth. This shows that $\bar{X}^{\varepsilon}(D)$ has potentially good reduction at $\wp$.

Lemma 10.3. The family $\bar{f}: \bar{X}(13) \rightarrow \bar{C}(13)$ does not have potentially good reduction at $p=3$.

Proof. Let $D=13$ and $p=3$. We compute the stable model of the generic fiber of $\bar{f}(13)$. Let $\mathcal{R}$ be the completion of $\mathbb{Z}[\sqrt{13}](t)$ at a prime above $p=3$. We let $X_{3}$ be the fiber of $\bar{X}(13)$ above the generic point of $\bar{C}(13)$. We claim that, after replacing $\mathcal{R}$ by the completion of a finite extension, there exists a stable model of $X_{3}$ over $\operatorname{Spec}(\mathcal{R})$ whose special fiber consists of two elliptic curves intersecting in one point. This follows by explicitly blowing up the equation of the curve $X_{3}$, as in the proof of Proposition 10.2, The lemma follows from this and the uniqueness of the stable model.

It is interesting that in the case that $D=13$ there is another prime besides $D$ and 2 which is exceptional for any choice of the model of $x_{D}$.

Question 10.4. Is there a model of $X_{D}$ such that $\mathcal{S}$ is minimal and if yes, what is this set $\mathcal{S}$ ? Is there a canonical model of $X_{D}$, comparable to the one for Shimura curves?

\section{REFERENCES}

[1] Y. André. G-functions and geometry. Aspects of Mathematics 13, Vieweg, 1989.

[2] Y. André. Sur la conjecture des $p$-courbures de Grothendieck-Katz et un problème de Dwork. In Geometric aspects of Dwork theory, 55-112, Walter de Gruyter GmbH \& Co. KG, 2004.

[3] M. Bainbridge. Euler characteristics of Teichmüller curves in genus two. Geom. Topology, 11:18872074, 2007.

[4] J. Bertin, J-P. Demailly and L. Illusie. Introduction à la théorie de Hodge. Panoramas et Synthèses 3 , Société Mathématique de France, 1996.

[5] F. Beukers and J. Stienstra. On the Picard-Fuchs equation and the formal Brauer group of certain elliptic K3-surfaces. Math. Ann., 271:269-304, 1985.

[6] F. Beukers. Gauss' hypergeometric function. In Arithmetic and geometry around hypergeometric functions, Progr. Math. 260, 23-42, Birkhäuser, 2007.

[7] F. Beukers. On Dwork's accessory parameter problem. Math. Z., 241:425-444, 2002.

[8] I.I. Bouw. The p-rank of curves and covers of curves. In: Courbes semi-stables et groupe fondamental en géométrie algébrique, Progr. Math. 187, 267-277, Birkhäuser, 2000.

[9] I.I. Bouw and M. Möller. Teichmüller curves, triangle groups, and Lyapunov exponents. arXiv:math/0511738, 2005.

[10] F. Catanese and R. Pignatelli. Fibrations of low genus, I. Ann. Sci. Éc. Norm. Sup., 39:1011-1049, 2006.

[11] D.V. Chudnovsky and G.V. Chudnovsky. Computer algebra in the service of mathematical physics and number theory. In Computers in mathematics, Lecture Notes in Pure and Appl. Math. 125, 109-232, 1990.

[12] B. Dwork. Differential operators with nilpotent p-curvature. Amer. J. Math., 112:749-786, 1990.

[13] N. Elkies. Shimura curve computations. In Algorithmic number theory, Lecture Notes in Comput. Sci. 1423, 1-47, 1998.

[14] T. Honda. Algebraic differential equations. In Symposia Mathematica, Vol. XXIV, 169-204, Academic Press, 1981.

[15] N.M. Katz. Nilpotent connections and the monodromy theorem: applications of a result of Turrittin. Inst. Hautes Études Sci. Publ. Math., 39:355-412, 1970.

[16] N.M. Katz. Expansion-coefficients as approximate solution of differential equations. Astérisque, 119120:183-189, 1984. 
[17] M. Kontsevich and D. Zagier. Periods. In Mathematics unlimited-2001 and beyond, 771-808, Springer, 2001.

[18] D. Krammer. An example of an arithmetic Fuchsian group. J. reine angew. Math., 473:69-85, 1996.

[19] G. Margulis. Discrete subgroups of semisimple Lie groups. Ergebnisse der Mathematik und ihre Grensgebiete 17, Springer, 1991.

[20] H. Masur. On a class of geodesics in Teichmüller space. Annals of Math., 102:205-221, 1975.

[21] C. McMullen. Billiards and Teichmüller curves on Hilbert modular sufaces. J. Amer. Math. Soc., 16:857-885, 2003.

[22] C. McMullen. Teichmüller curves in genus two: Discriminant and spin. Math. Ann., 333:87-130, 2005.

[23] C. McMullen. Teichmüller curves in genus two: Torsion divisors and ratios of sines. Invent. Math., 165:651-672, 2006.

[24] M. Möller. Variations of Hodge structures of Teichmüller curves. J. Amer. Math. Soc., 19:327-344, 2006.

[25] M. Möller. Finiteness results for Teichmüller curves. To appear in Ann Inst. Fourier.

[26] R. Silhol. Genus 2 translation surfaces with an order 4 automorphism. In: The geometry of Riemann surfaces and abelian varieties, Contemp. Math. 397, 207-213, Amer. Math. Soc., 2006.

[27] W. Veech. Teichmüller curves in moduli space, Eisenstein series and an application to triangular billiards. Invent. Math., 97:533-583, 1989.

[28] M. Yoshida. Fuchsian differential equations., Aspects of Math. 11, Vieweg, 1987.

Irene I. Bouw

Institut für reine Mathematik

Helmholtzstraße 18

89069 Ulm

irene.bouw@uni-ulm.de
Martin Möller

Max-Planck-Institut für Mathematik

Vivatsgasse 7

53111 Bonn

moeller@mpim-bonn.mpg.de 